\title{
Wideband Cognitive Radio Networks Based Compressed Spectrum Sensing: A Survey
}

\author{
Mohammed Abo-Zahhad1,2, Sabah M. Ahmed ${ }^{2,3}$, Mohammed Farrag2,4, Khaled Ali BaAli ${ }^{2,5}$ \\ ${ }^{1}$ Electronics and Communication Engineering Department, School of Electronics, Communications and Computer Engineering, \\ Egypt-Japan University for Science and Technology, Alexandria, Egypt \\ ${ }^{2}$ Electrical and Electronics Engineering Department, Faculty of Engineering, Assiut University, Assiut, Egypt \\ ${ }^{3}$ Mechatronics and Robotics Engineering Department, School of Innovative Design Engineering, Egypt-Japan University for \\ Science and Technology, Alexandria, Egypt \\ ${ }^{4}$ Electrical Engineering Department, King Khalid University (KKU), Abha, KSA \\ ${ }^{5}$ Electronics and Communication Department, Faculty of Engineering and Petroleum, Hadhramaut University, Mukalla, \\ Republic of Yemen \\ Email: mohammed.zahhad@ejust.edu.eg, sabah.ahmed@ejust.edu.eg,mfarrag@kku.edu.sa,khaled_baali@eng.au.edu.eg
}

How to cite this paper: Abo-Zahhad, M., Ahmed, S.M., Farrag, M. and BaAli, K.A. (2018) Wideband Cognitive Radio Networks Based Compressed Spectrum Sensing: A Survey. Journal of Signal and Information Processing, 9, 122-151.

https://doi.org/10.4236/jsip.2018.92008

Received: March 18, 2018

Accepted: May 28, 2018

Published: May 31, 2018

Copyright $(9) 2018$ by authors and Scientific Research Publishing Inc. This work is licensed under the Creative Commons Attribution International License (CC BY 4.0).

http://creativecommons.org/licenses/by/4.0/

\begin{abstract}
Spectrum sensing is a core function at cognitive radio systems to have spectrum awareness. This could be achieved by collecting samples from the frequency band under observation to make a conclusion whether the band is occupied, or it is a spectrum hole. The task of sensing is becoming more challenging especially at wideband spectrum scenario. The difficulty is due to conventional sampling rate theory which makes it infeasible to sample such very wide range of frequencies and the technical requirements are very costly. Recently, compressive sensing introduced itself as a pioneer solution that relaxed the wideband sampling rate requirements. It showed the ability to sample a signal below the Nyquist sampling rate and reconstructed it using very few measurements. In this paper, we discuss the approaches used for solving compressed spectrum sensing problem for wideband cognitive radio networks and how the problem is formulated and rendered to improve the detection performance.
\end{abstract}

\section{Keywords}

Cognitive Radio, Spectrum Sensing, Compressive Sensing, Compressed Measurements, Nyquist Sampling Rate

\section{Introduction}

The spectrum scarcity has created a false believe that there are no more useful 
radio frequencies left. This false believe occurred due to the current spectrum allocation policy and made the spectrum underutilization, i.e., nearly $70 \%$ of radio occupancy was not used most of the time [1] [2]. The problem of underutilization led to have many frequency bands not operative or Spectrum Holes (SHs). SHs are not but frequency bands assigned to Primary Users (PUs) at specific frequencies and geographic locations not used for period. So, to solve the spectrum issue, the Federal Communications Commission (FCC) suggested opening these frequency bands dedicated to the PUs to be shared with Secondary Users (SUs). Opening these frequency bands is known later as Dynamic Spectrum Access (DSA) [3] [4]. In the DSA, the SUs access these unoccupied bands whenever the PUs are absent and should terminate their transmission when PUs transmitted signals are detected. Also, the SUs should keep the inference background within acceptable limit that does no harm to the communication transmissions of both PUs and other SUs. Figure 1 manifests that certain lot of the radio bands is sparsely used and substantial amount of these bands is unutilized [2].

However, a question may pose; how do SUs identify these hidden spectral opportunities or SHs? Simply without complication by searching the spectrum looking for empty slots/bands or detecting primary user transmitted signal on

\section{Measured Spectrum Occupancy in Chicago, IL}

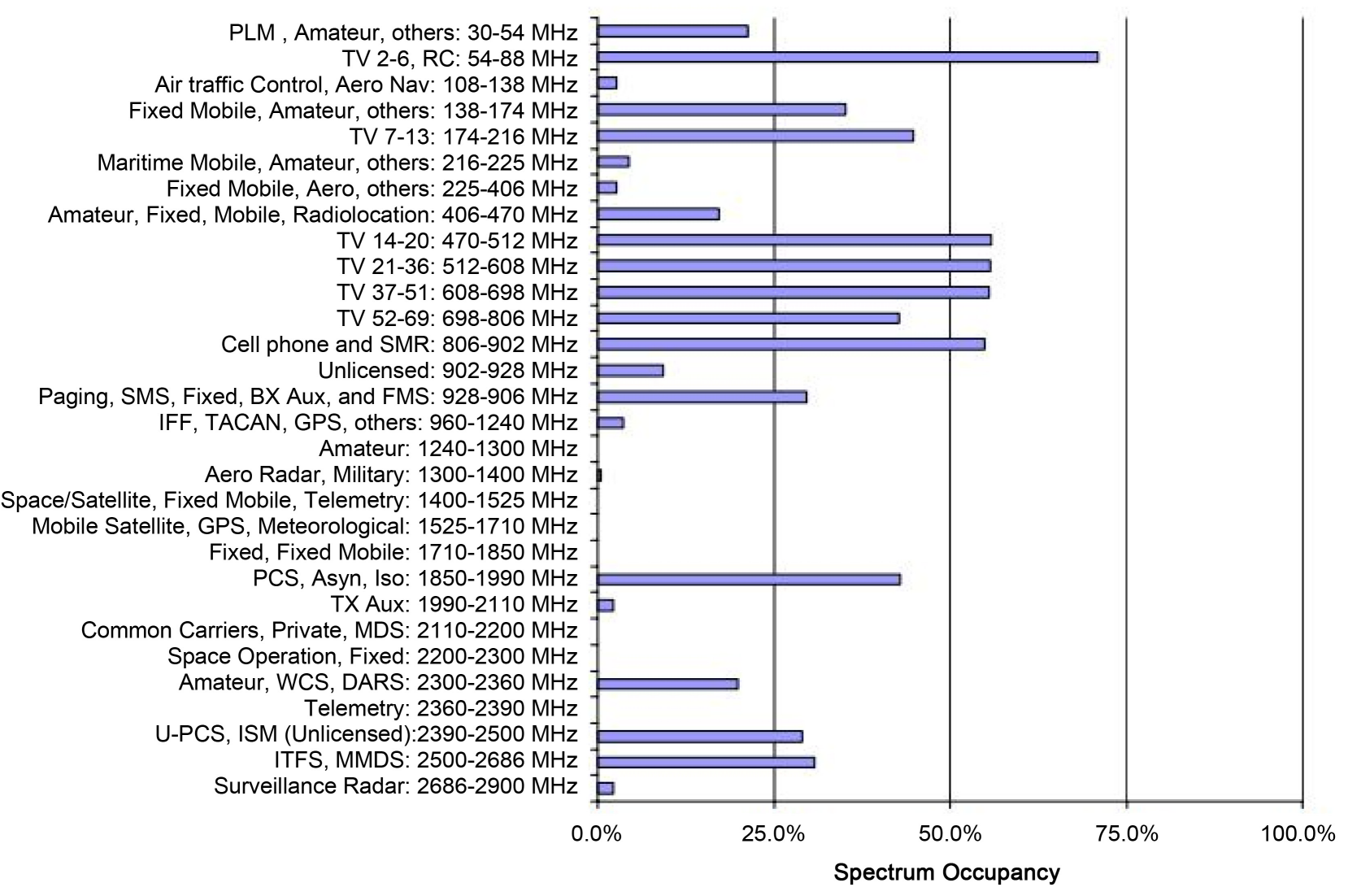

Figure 1. Spectrum occupancy in each band measured in Chicago. 
the band(s) under observation. For more clarity, spectrum sensing mechanism is nothing but collecting samples from the frequency band(s) of interest, then thresholding and draw up a conclusion about that band(s) statue [5]. Cognitive Radio (CR) as DSA technique enables SUs the spectrum awareness. This arises from that it has the ability to adapt its transmission parameters to meet spectral opportunities requirements such as transmission power, modulation schemes, code rates, etc. [4] [6] [7] [8].

Spectrum sensing is a mandatory component at IEEE 802.22 WRAN standardization to enable self-coexistence for $\mathrm{CR}$ and it could be achieved by two methods [9] [10]. In the first method, the radio itself becomes spectrum awareness by detecting and estimating the spectrum to identify which channels are occupied and which are unoccupied [11]. In the second method, geolocation and database or beacon are used for identifying SHs [9]. However, the second method requires the involvement of PUs cooperation which violates the condition of CR's independency on performing sensing task depending on its own ability without PU assistance. Moreover, spectrum sensing performance could be improved if cooperation between SUs is involved to mitigate hidden node problem, multipath fading and shadowing [12] [13]. And even further enhanced performance could be achieved by introducing Compressive Sensing (CS) technique. Most of researches focused on detecting primary transmitter signal rather than primary receiver detection due to some technical difficulties with the latter.

The sensing task becomes more challenging especially in wideband spectrum [14], e.g., a range up to $20 \mathrm{GHz}$. Conventional Digital Signal Processing (DSP) may take long time processing this wide range of frequencies and it's very expensive. To the best of our knowledge, the achievable sampling rate of the current Analog-to-digital convertor (ADC) is 3.6 Giga Sample per second or 3.6 GSps [15]. Filter bank technique could ease sampling requirements by turn wideband into narrowband [16] but we end with another problem which is the number of filters required. Nyquist sampling rate is very serious problem in wideband spectrum once it is rendered the rest is straight forward task. CS is a powerful tool that cracked the sampling rate problem [17]. It proved the possibility of sampling below Nyquist sampling rate, also known as sub-Nyquist sampling rate, and reconstructed the transmitted signal after sub-Nyquist sampling. However, it requires the signal to be sparse or compressible [18] [19]. If a signal is not sparse, it may transform to another domain at which sparsity condition is hold. Numerous research papers were published to identify spectrum holes-based CS through signal reconstruction algorithms. This kind of spectrum holes identification dominant since then. To the best of our knowledge there are few publications that concern with spectrum sensing problem as detection problem without going to reconstruction stage [20] [21] [22].

In general, spectrum sensing problem can be discussed in several ways based on different criterions and different assumptions. For example, if the classification is based on what or how to sense, then discussion would classify the spec- 
trum sensing to:1) transmitter detection, 2) cooperative detection, and 3) interference-based detection [11]. Depending on the type of applications leads to highlight to the spectrum types, either narrow band sensing or wideband sensing. Proactive and reactive sensing are another aspect of classification. Mostly, in literature, spectrum sensing is based on transmitter detection due infeasibility to detect primary receiver signal such as the location of PU receiver [6] [11]. Different detection techniques, i.e., energy detection, matched filter, cyclostationary detection, covariance-based detection wavelet, compressed detection..., etc, can be employed to detect the SHs. Spectrum sensing framework is depicted in Figure 2. Comparison of the main spectrum sensing is illustrated in Table 1.

Even though there are numerous research papers that discussed the problem of spectrum sensing for wideband cognitive radio based compressed sensing, nevertheless, the detection problem is formulated as detection based-signal reconstruction using compressed sensing which is the prevailing trend [5] [6] [18] [23]. In [23], the authors have focused on discussing technical implementation issues of Analog to Information Conversion (AIC). In this paper the compressed spectrum sensing problem at wideband CR networks is addressed. And the recent research achievements in solving the problem are discussed, e.g., how the problem is formulated and rendered to improve the detection performance.

The rest of this paper is structured as follows. Section 2 highlighted the wideband CR spectrum sensing challenge. In Section 3, compressed spectrum sensing problem is formulated. Compressed wideband CR spectrum detection approaches are discussed in Section 4. The open research problems of the wideband CR networks based compressive spectrum sensing are introduced in Section 5. Finally, Section 6 presents the main conclusions.

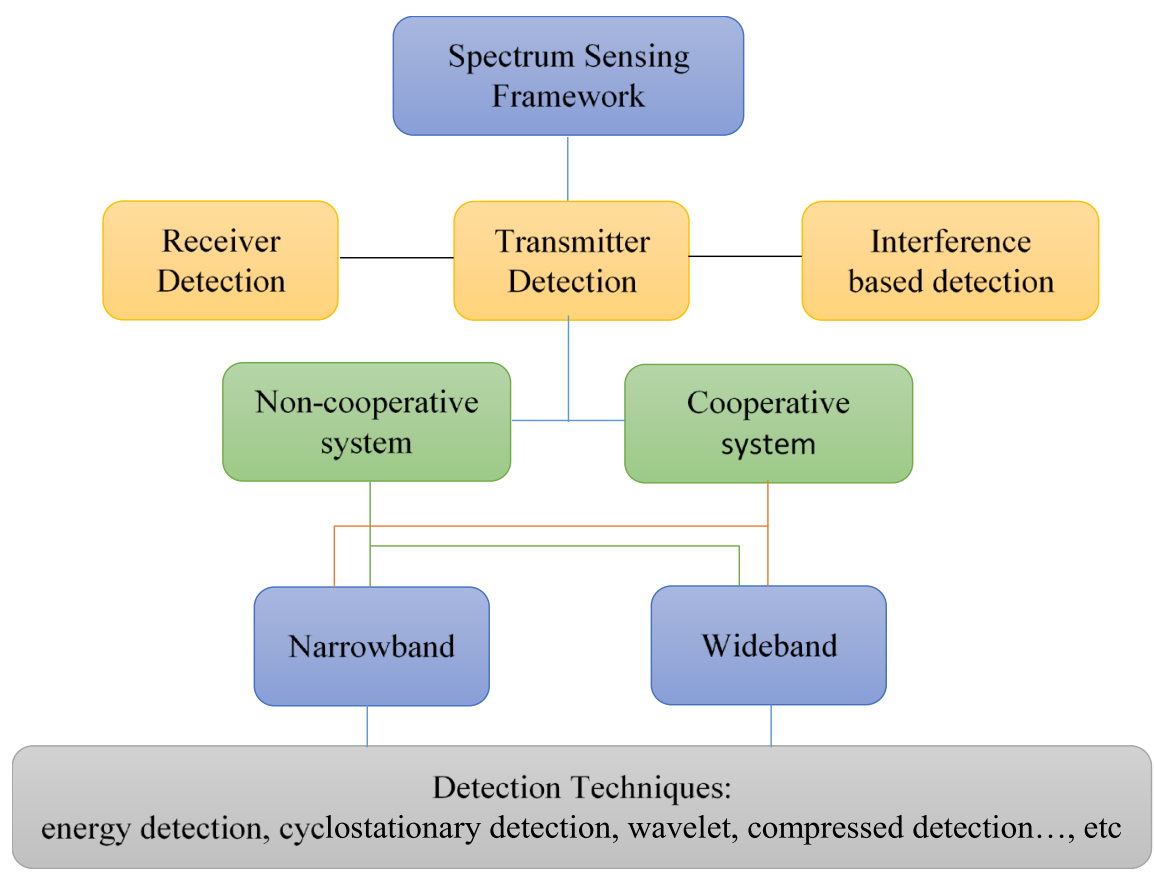

Figure 2. Spectrum sensing framework. 
Table 1. Comparison of main spectrum sensing techniques.

\begin{tabular}{|c|c|c|c|}
\hline \multirow{2}{*}{$\begin{array}{l}\text { Detection } \\
\text { technique }\end{array}$} & \multicolumn{3}{|c|}{ Performance metrics } \\
\hline & Accuracy & Complexity & Robustness \\
\hline Energy detection & $\begin{array}{l}\text { - at high SNRs, the } \\
\text { performance is } \\
\text { good } \\
\text { - at low SNRs, the } \\
\text { performance is } \\
\text { unreliable } \\
\text { - } \quad \text { affected by noise } \\
\text { uncertainty }\end{array}$ & $\begin{array}{ll}\text { - } & \text { Low } \\
\text { implementation } \\
\text { complexity } \\
\text { - } & \text { convergence is } \\
\text { reach by collecting } \\
\text { higher number of } \\
\text { samples }\end{array}$ & $\begin{array}{ll}\text { - } & \text { orior information } \\
\text { of PU signal isn't } \\
\text { required } \\
\text { - } & \text { inappropriate for } \\
\text { spread spectrum } \\
\text { signals } \\
\text { - } & \text { cannot } \\
\text { differentiate a PU } \\
\text { signal from other } \\
\text { signal sources }\end{array}$ \\
\hline Feature detection & $\begin{array}{l}\text { - the performance } \\
\text { is good at all } \\
\text { SNRs }\end{array}$ & $\begin{array}{ll}\text { - } & \text { medium } \\
\text { complexity } \\
\text { - } & \text { convergence re- } \\
\text { quires small num- } \\
\text { ber of samples }\end{array}$ & $\begin{array}{ll}\text { - } & \text { require partial } \\
\text { knowledge of } \\
\text { PU signal } \\
\text { - } \quad \text { robust against } \\
\text { noise uncertainty } \\
\text { and interference } \\
\text { - } & \text { differentiate PU } \\
& \text { signal among } \\
\text { different types of } \\
\text { signal source }\end{array}$ \\
\hline $\begin{array}{c}\text { Matched filter } \\
\text { and coherent } \\
\text { detection }\end{array}$ & $\begin{array}{l}\text { - best performance } \\
\text { at all SNRs }\end{array}$ & $\begin{array}{ll}\text { - } & \text { high complexity } \\
\text { - convergence } \\
\text { requires fewest } \\
\text { number of samples }\end{array}$ & 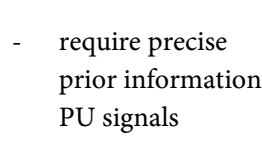 \\
\hline $\begin{array}{l}\text { Covariance-based } \\
\text { detection }\end{array}$ & $\begin{array}{l}\text { - detection } \\
\text { accuracy is high }\end{array}$ & $\begin{array}{l}\text { - low computational } \\
\text { complexity }\end{array}$ & 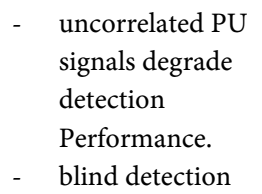 \\
\hline
\end{tabular}

\section{Wideband CR Spectrum Sensing Challenge}

In literature, there have been several signal processing challenges facing CR since certain tasks have become more complicated. Some of these were discussed in [6] [24]. In this section, we discuss the wideband spectrum sensing problem in the sense that the complication in this type of spectrum is very high. In fact, there is no signal processing approach yet exist that could provide the locations of all the hidden spectral opportunities and at what frequency band at one single shot. Several technical challenges are facing wideband sensing and limiting its operation. One of these challenges is the need for high data rate radio front end requirement to sense a wide frequency range. This adds additional constraint for CR system deployment especially on units with limited data rate processors. Conventional estimation methods based on Nyquist sampling are challenging task in wideband spectrum. Even though using filter banks may help to turn a wideband signal into narrowband to be easy sensed using conventional methods, but with the cost of using large number of narrowband filters. Even though the 
above-mentioned problem is very challenging in wideband CR spectrum sensing, we will see how compressive sensing technique relaxes Nyquist sampling rate effectively alongside with reducing both complexity and power consumption requirements.

\section{Compressive Spectrum Sensing Problem}

The idea of using learning and sensing machines to probe the radio spectrum was envisioned several decades earlier. Two different frameworks regarding how to formulate spectrum sensing were presented. Signal detection and signal classification which encapsulated the spectrum sensing on coming up a decision whether the PU transmitted signal, e.g., deterministic or random, is present or not from the observed received signals [25]. Due to the importance of this topic, we will discuss the sensing or detection problem as entrance to compressive spectrum sensing problem template. Moreover, we will investigate how the detection based compressive sensing could improve the detection performance in CR systems.

\subsection{Signal Sensing Problem}

Signal detection has been an important topic in wireless communication engineering and in CR. It is considered as a core function. In CR the radio frequency spectrum is sensed to come out with a decision whether the PU transmitted signal, mostly very weak PU signals, exists in a certain frequency band of interest or not. In other words, it enables the SUs identifying the SHs. So, we could formulate the spectrum sensing problem by the classical binary hypothesis test [5] [11] [26] as follows:

$$
x(t)=\left\{\begin{array}{l}
n(t): \mathcal{H}_{0}, \mathrm{PU} \text { is absent } \\
s(t)+n(t): \mathcal{H}_{1}, \mathrm{PU} \text { is present }
\end{array}\right.
$$

where, $x(t)$ is the received signal at the $\mathrm{SU}$ receiver during observation window T. $n(t)$ is the additive white Gaussian noise (AWGN) with zero mean and variance $\sigma^{2}$, i.e., $n \sim \mathcal{N}\left(0, \sigma^{2}\right) . \sigma^{2}$ is also considered as a noise power. $s(t)$ is the transmitted PU signal and it is independent and identical Gaussian random variable. $\mathcal{H}_{0}$ and $\mathcal{H}_{1}$ denote the absence and the presence of the PU respectively. The decision between the two hypotheses is based on the comparison of statistical test to a predetermined threshold $\lambda$ as follows;

$$
T \underset{\mathcal{H}_{0}}{\stackrel{\mathcal{H}_{1}}{\gtrless}} \lambda
$$

where, $T$ is the statistical test generated to decide if the observed signal $x(t)$ under hypothesis $\mathcal{H}_{0}$ or $\mathcal{H}_{1}$. Most widely used detectors, e.g., energy detection, and cyclostationary detection, perform the statistical test to differentiate the PU transmitted signal from noise as depicted in Figure 3.

In the context of spectrum sensing, the performance of sensing is measured by the Receiver Operating Characteristics (ROC) curves. It is a tradeoff between 


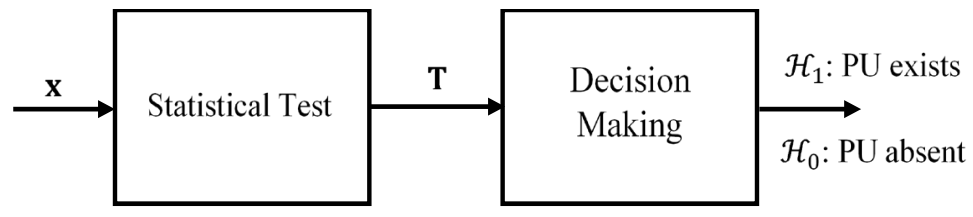

Figure 3. Primary user signal detection scenario.

probability of detection versus probability of false alarm or probability of miss-detection versus probability of false alarm [26]. Let us assume the received signal in (1) follows normal Gaussian distribution as depicted in Figure 4. Then then decision statistics under each hypothesis $\mathcal{H}_{0}$ and $\mathcal{H}_{1}$ could be denoted as $f\left(\mathbf{x} \mid \mathcal{H}_{0}\right)$ and $f\left(\mathbf{x} \mid \mathcal{H}_{1}\right)$, respectively.

If the observed sample of $x(t)$ is greater than the threshold, the detector is more likely to choose $\mathcal{H}_{1}$ or $f\left(\mathbf{x} \mid \mathcal{H}_{1}\right)>f\left(\mathbf{x} \mid \mathcal{H}_{0}\right)$. The error in this scheme occurs as follows; if the detector decides $\mathcal{H}_{1}$ when $\mathcal{H}_{0}$ true and this type of error is known as false alarm. On the other hands, if the detector decides $\mathcal{H}_{0}$ when $\mathcal{H}_{1}$ true and this type of error is known as miss detection. These two errors are inevitable to some extent nevertheless may be traded off against each other to achieve high probability of detection $\mathrm{P}\left(\mathcal{H}_{1} \mid \mathcal{H}_{1}\right)$ to guarantee minimal interference with the PU [26].

At the same time, we need to have a low probability of false alarm $\mathrm{P}\left(\mathcal{H}_{1} \mid \mathcal{H}_{0}\right)$ to improve the SU throughput. This setup is called the Neyman-Pearson (NP) approach to spectrum sensing. The reason of using NP test is we do not know the prior probabilities for $\mathrm{P}\left(\mathcal{H}_{0}\right)$ and $\mathrm{P}\left(\mathcal{H}_{1}\right)$ which yields having a problem in minimizing the averaging probability of error. Also, we could not make risk minimization which requires having the prior probabilities of $\mathcal{H}_{0}$ and $\mathcal{H}_{1}$. Maximum likelihood is an optimal detector but we have no way to evaluate its performance for the same problem of not having any knowledge of the prior probabilities [5]. The performance of the detector will be evaluated in term of these probabilities of detection, miss detection and false alarm, defined as;

Probability of detection $\left(P_{D}\right)$ : it is the probability when SU detects PU transmitted signal correctly or declaring $\mathcal{H}_{1}$ under $\mathcal{H}_{1}, \mathrm{P}\left(\mathcal{H}_{1} \mid \mathcal{H}_{1}\right)$.

$$
P_{D}=\mathrm{P}\left(\text { signal is detected } \mid \mathcal{H}_{1}\right)=\mathrm{P}\left(X>\lambda \mid \mathcal{H}_{1}\right)
$$

Probability of false alarm $\left(P_{F}\right)$ : it is the probability when SU declares that $\mathrm{PU}$ is present, when it is not or declaring $\mathcal{H}_{1}$ under $\mathcal{H}_{0}, \mathrm{P}\left(\mathcal{H}_{1} \mid \mathcal{H}_{0}\right)$.

$$
P_{\mathrm{F}}=\mathrm{P}\left(\text { signal is detected } \mid \mathcal{H}_{0}\right)=\mathrm{P}\left(X>\lambda \mid \mathcal{H}_{0}\right)=\int_{\lambda}^{\infty} f\left(\boldsymbol{x} \mid \mathcal{H}_{0}\right) \mathrm{d} x
$$

Probability of miss-detection $\left(P_{M}\right)$ : it is probability of failing to detect the $\mathrm{PU}$ presence when it is there or declaring $\mathcal{H}_{0}$ under $\mathcal{H}_{1}, \mathrm{P}\left(\mathcal{H}_{0} \mid \mathcal{H}_{1}\right)$.

$$
P_{M}=\mathrm{P}\left(\text { signal is not detected } \mid \mathcal{H}_{1}\right)=\mathrm{P}\left(X>\lambda \mid \mathcal{H}_{1}\right)
$$

\subsubsection{Probability of Detection and False Alarm over AWGN Channels}

The spectrum sensing problem could be formulated as a constrain optimization problem; maximize $P_{D}$ where $\alpha$ is a specified maximum tolerable false alarm 


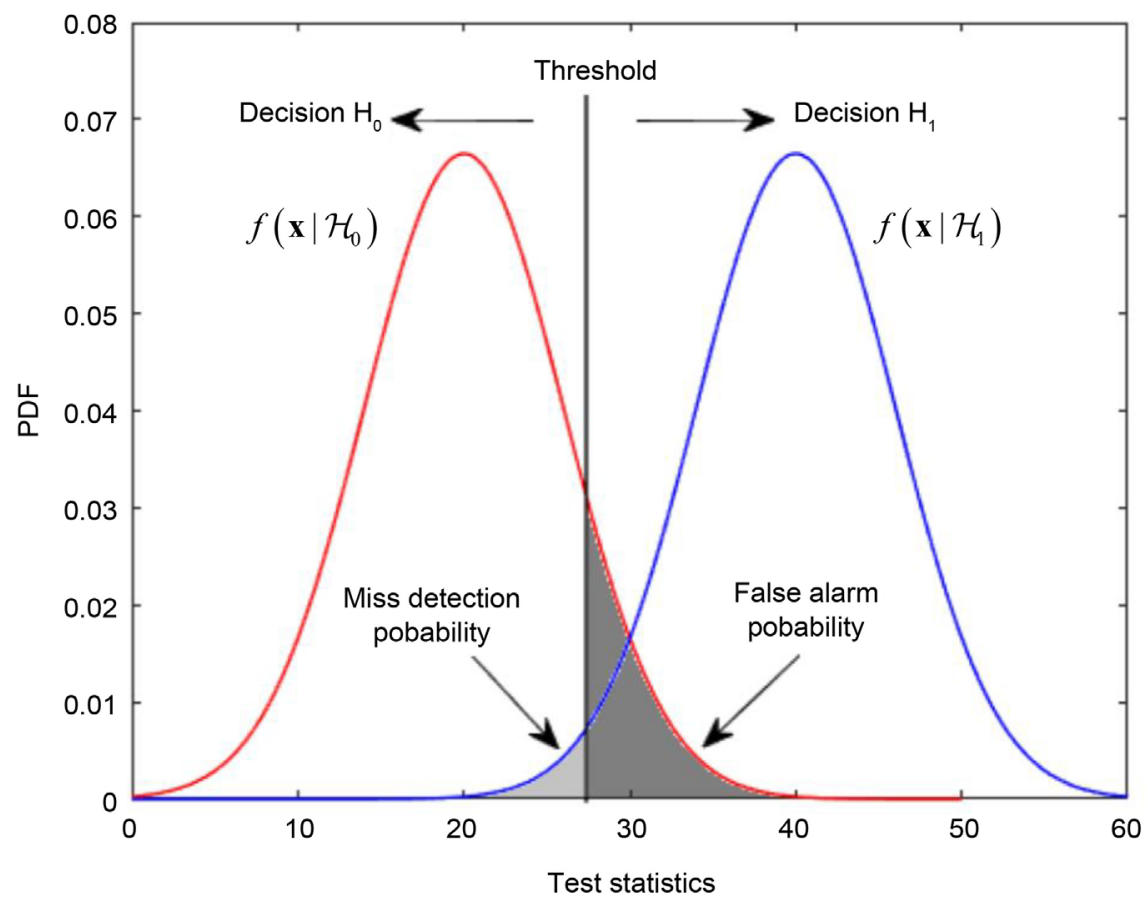

Figure 4. Trade off error by adjusting threshold.

probability [26]. In summary, ROC curves allow us to have insight understanding to that govern the relationship between the sensitivity $\left(P_{D}\right)$ and specificity $\left(P_{F}\right)$ of a spectrum sensing scheme for a variety of different algorithm parameters [27]. If the observed signal in (1) is deterministic, the test statistics could then be modeled as [28]

$$
T \sim\left\{\begin{array}{l}
N\left(0, \sigma^{2}\|s\|^{2}\right): \mathcal{H}_{0} \\
N\left(\|s\|^{2}, \sigma^{2}\|s\|^{2}\right): \mathcal{H}_{1}
\end{array}\right.
$$

And by solving (3) and (4), we have

$$
\begin{gathered}
P_{F}=\int_{\lambda}^{\infty} f\left(x \mid H_{1}\right) \mathrm{d} x=\int_{\lambda}^{\infty} \frac{1}{\sqrt{2 \pi \sigma^{2}}} \exp \left(\frac{\|s\|^{2}}{2 \sigma^{2}}\right) \mathrm{d} x=Q\left(-\frac{\lambda}{\sqrt{\sigma^{2}\|s\|^{2}}}\right) \\
P_{D}=\int_{\lambda}^{\infty} f\left(x \mid H_{1}\right) \mathrm{d} x=\int_{\lambda}^{\infty} \frac{1}{\sqrt{2 \pi \sigma^{2}}} \exp \left(\frac{x-\|s\|^{2}}{2 \sigma^{2}}\right) \mathrm{d} x=Q\left(-\frac{\lambda-\|s\|^{2}}{\sqrt{\sigma^{2}\|s\|^{2}}}\right)
\end{gathered}
$$

where, $Q(\cdot)$ is the complementary distribution function of a standard Gaussian. For indirect method, we could specify the amount of threshold required to maximize the $P_{D}$ for certain $P_{F}$ from (7a) as follows

$$
\lambda=\sqrt{\sigma^{2}\|S\|^{2}} Q^{-1}\left(P_{F}\right)
$$

Substituting (8) into (7b), it yields

$$
P_{D}=Q\left(Q^{-1}\left(P_{F}\right)-\|x\| / \sqrt{\sigma^{2}}\right)=Q\left(Q^{-1}\left(P_{F}\right)-\sqrt{\gamma}\right)
$$


where, $\gamma=\|s\|^{2} / \sigma^{2}$, i.e., the signal-to-noise ratio SNR. On the other hand, if PU transmitted signal $s(t)$ assumed to be an unknown deterministic signal, the distribution of the decision statistics follows central chi-square $\chi_{N}^{2}$ under $\mathcal{H}_{0}$ and non-central chi-square $\chi_{N}^{\prime 2}$ with $T W$ degrees of freedom under $\mathcal{H}_{1}$ [29]. However, for the sake of simplicity, we exchange the time-bandwidth product by an integer number $N$. In literature, some researchers used instead of $T W$, they used $2 T W(N \rightarrow 2 N)$. In other words, we could use either even or odd degrees of freedom for the analytic processes relying on the type of applications.

$$
\zeta \sim\left\{\begin{array}{l}
\chi_{N}^{2}: \mathcal{H}_{0} \\
\chi_{N}^{\prime 2}: \mathcal{H}_{1}
\end{array}\right.
$$

The probability density function of the decision statistics $X_{i}$ is specified by

$$
f_{x}(x)=\left\{\begin{array}{l}
\frac{1}{\sigma^{2} 2^{N / 2} \Gamma(N / 2)} x^{(N / 2-1)} \exp \left(-x / \sigma^{2}\right): \mathcal{H}_{0} \\
\frac{1}{2}\left(\frac{x}{\mu}\right)^{(N-2 / 4)} \exp (-\mu+x / 2) I_{N / 2-1}(\sqrt{\mu x}): \mathcal{H}_{1}
\end{array}\right.
$$

where, $\mu$ is the noncentrality parameter $\left(\mu \propto\|s\|^{2} / \sigma^{2}\right)$ and $I_{v-1}(\cdot)$ is the $v$-th modified Bessel function of the first kind. The $P_{F}$ and $P_{D}$ over AWGN is given respectively by:

$$
\begin{gathered}
P_{F}=\operatorname{Pr}\left(x>\lambda \mid \mathcal{H}_{0}\right)=\int_{\lambda}^{\infty} f_{x, \mathcal{H}_{0}}(x) \mathrm{d} x \\
=\int_{\lambda}^{\infty} \frac{1}{\sigma^{2} 2^{N / 2} \Gamma(N / 2)} x^{(N / 2-1)} \exp \left(-x / \sigma^{2}\right) \mathrm{d} x \\
P_{F}=\Gamma\left(N / 2, \lambda / 2 \sigma^{2}\right) / \Gamma(N / 2)
\end{gathered}
$$

Similarly,

$$
\begin{gathered}
P_{D}=\operatorname{Pr}\left(x>\lambda \mid \mathcal{H}_{1}\right)=\int_{\lambda}^{\infty} f_{x, \mathcal{H}_{1}}(x) \mathrm{d} x \\
=\int_{\lambda}^{\infty} \frac{1}{2}\left(\frac{x}{\mu}\right)^{(N-2 / 4)} \exp (-\mu+x / 2) I_{N / 2-1}(\sqrt{\mu x}) \mathrm{d} x \\
P_{D}=Q_{\frac{N}{2}}\left(\sqrt{\mu / \sigma^{2}}, \sqrt{\lambda / \sigma^{2}}\right)
\end{gathered}
$$

where, $\Gamma(\cdot, \cdot), \Gamma(\cdot)$, and $Q_{v}(\cdot, \cdot)$ are complete and in complete gamma function and generalized Marcum Q-function, respectively. As the number of degrees of freedom increases, the $\chi_{N}^{2}$ and $\chi_{N}^{\prime 2}$ probability density functions asymptotically turn out to be Gaussian by the central limit theorem [28].

$$
P D=Q\left(Q^{-1}(P F)-\sqrt{N} \gamma\right)
$$

A board published research has been conducted and discussed spectrum sensing in CR. To the best of our knowledge most of the researches are dedicated to improving the detection performance of the energy detector. Since it does not involve complicated signal processing and has short time response and low complexity cost [30]. It is called blind technique in that it does not require prior 
information about the PU transmitted signal. Despite these merits made it preferable at wide variety of applications such as CR systems and ultra-wideband communications, it is subjected to noise uncertainty issue. On the other hand, cyclostationary detectors outperform all other detectors in term of spectrum sensing performance but with the expense of computational complexity. However, it requires long sensing time. Table 2 includes some closed forms of spectrum sensing performance based on computations of $P_{D}, P_{F}$ and $P_{M}$, where, $E$ is the received signal power.

\subsubsection{Probability of Detection and False Alarm over Fading Channels}

In the proceeding section, the spectrum sensing performance over AWGN channel and the derived detection probabilities pairs $\left(P_{D}, P_{F}\right)$ have been discussed. To broad the picture for more than talking on AWGN, fading channel should not be ignored, e.g., Rayleigh, Nakagami-m, lognormal,..., etc. The analytical expression $P_{D}$ could be undergone of two popular methods; namely, the probability density function and the moment generating function (MGF) relying on the fading channel statistical characteristic to derive the performance analysis [31]. However, the MGF method is restricted to integer-valued fading descriptors. The conventional method for obtaining the average $P_{D}$ over specific fading distribution is by averaging $P_{D}$ of AWGN over $f_{\gamma}(\gamma)$ of the output SNR. This method could be expressed as:

$$
\bar{P}_{D}=\int_{0}^{\infty} P_{D}(\gamma) f_{\gamma}(\gamma) \mathrm{d} \gamma
$$

where $f_{\gamma}(\gamma)$ is the fading channel distribution. Alternatively, it could be expressed by:

$$
\bar{P}_{D}=\int_{0}^{\infty} Q_{\frac{N}{2}}\left(\sqrt{\mu / \sigma^{2}}, \sqrt{\lambda / \sigma^{2}}\right) f_{\gamma}(\lambda) \mathrm{d} \gamma
$$

with $\mu \propto \gamma(\mu=\alpha \gamma)$ and $\alpha$ is a positive value. Many channel models have been suggested to define the amplitude and phase of multipath fading signals statistics. Among them Nakagami-m distribution is a generalized distribution to model different fading environments [32]. It has greater flexibility and accuracy

Table 2. Probabilities of detection and false alarm of well-known detectors over AWGN.

\begin{tabular}{cccc}
\hline Detector type & $\begin{array}{c}\text { Probability of } \\
\text { detection }\end{array}$ & $\begin{array}{c}\text { Probability of false } \\
\text { alarm }\end{array}$ & $\begin{array}{c}\text { Probability of } \\
\text { miss-detection }\end{array}$ \\
\hline $\begin{array}{c}\text { Matched } \\
\text { filtering }\end{array}$ & $Q\left(\sqrt{\frac{2 E}{\sigma^{2}}}, \sqrt{\frac{2 \lambda^{2}}{E \sigma^{2}}}\right)$ & $\exp \left(-\frac{\lambda^{2}}{E \sigma^{2}}\right)$ & $1-Q\left(\sqrt{\frac{2 E}{\sigma^{2}}}, \sqrt{\frac{2 \lambda^{2}}{E \sigma^{2}}}\right)$ \\
Energy & $Q_{\frac{N}{2}\left(\sqrt{\frac{\mu}{\sigma^{2}}}, \sqrt{\frac{\lambda}{\sigma^{2}}}\right)}$ & $\frac{\Gamma\left(\frac{N}{2}, \frac{\lambda}{2 \sigma^{2}}\right)}{\Gamma\left(\frac{N}{2}\right)}$ & $1-Q_{\frac{N}{2}}\left(\sqrt{\frac{\mu}{\sigma^{2}}}, \sqrt{\frac{\lambda}{\sigma^{2}}}\right)$ \\
cyclostationary & $Q\left(\frac{\sqrt{2 \gamma}}{\sigma}, \frac{(2 N+1) \lambda}{\sigma^{2}}\right)$ & $\exp \left(-\frac{(2 N+1) \lambda^{2}}{E \sigma^{2}}\right)$ & $1-Q\left(\frac{\sqrt{2 \gamma}}{\sigma}, \frac{(2 N+1) \lambda}{\sigma^{2}}\right)$ \\
\hline
\end{tabular}


in matching some experimental data than the Rayleigh, lognormal or Rice distributions [31] [33]. The letter $-m$ in the word "Nakagami-m" is called Nakagami shape factor or the fading order. For example, $m=1$ is Rayleigh distribution which is sufficient to model amplitude in urban areas. While for $m>1$ Rician distribution suits better in sub-urban area where LOS components exist. Both Rayleigh and Rician distributions are special cases of Nakagami-m distribution. The problem of spectrum sensing over fading channels is addressed in [34]. And for sake of simplicity, we tabulate the closed forms obtained in Table 3 (after mathematical manipulations see [34]).

In the following section, the sensing problem in case of compressive sensing and its formulation will be discussed.

\subsection{Compressed Signal Detection Problem}

Compressed sensing proved the conventional sampling theory was not completely true [17] [35] [36]. In the sampling theory, the transmitted signal requires to be sampled, at least, twice the maximum frequency component in the signal to be able to reconstruct it successfully at the receiver side. In wideband spectrum scenario, the signal has $3 \mathrm{GHz}$ bandwidth and after sampling we get 6 $\mathrm{GHz}$ bandwidth which infeasible for implementation and signal processing. Even though current advance analog-to-digital conversion, to the best of our knowledge, achieve sampling rate at speed to $3.6 \mathrm{GSps}$ [15] but the cost of this signal processing is very high. CS showed that the possibility to sample below Nyquist sampling rate as long the signal is sparse or compressible [36]. We say that a certain signal is sparse if it contains few coefficients are nonzero and the rest are zeros. And the signal is compressible it has few large coefficients and the remainders are small or zero. Also, signal sparsity could be maintained by transformation, e.g., Fourier, DCT, wavelet, ..., etc. Signals with this type of representation yield to underdetermined system problem because we are seeking to reconstruct the transmitted signal with $N$ dimensions $\left(x \in \mathbb{R}^{N \times 1}\right)$ from the received signal with $m$ dimensions $\left(y \in \mathbb{R}^{m \times 1}\right)$ where, $m \ll N$. Mathematically

Table 3. Probability of detection over different types of fading channels.

\begin{tabular}{cc}
\hline $\begin{array}{c}\text { Type of fading } \\
\text { channel }\end{array}$ & Closed form expression of $\overline{P_{D}}$ \\
\hline Rayleigh & $\exp \left(-\lambda / 2 \sigma^{2}\right)[1+\eta]^{(N / 2-1)}\left[\exp \left(-\lambda / 2 \sigma^{2}\left(1+\eta^{2}\right)\right)-\sum_{k=0}^{N / 2-2} \frac{1}{k !}\left(\lambda / 2 \sigma^{2}\left(1+\eta^{2}\right)\right)^{k}\right]$ \\
& $+\exp \left(-\lambda / 2 \sigma^{2}\right) \sum_{k=0}^{N / 2-2} \frac{1}{k !}\left(\lambda / 2 \sigma^{2}\right)^{k}$ \\
Rician & $Q(\sqrt{2 K \bar{\gamma} / K+1+\bar{\gamma}}, \sqrt{\lambda(1+K) / K+1+\bar{\gamma}})$ \\
Nakagami-m & $1-(2 m / 2 m+\bar{\gamma})\left\{\bar{\gamma}\left(\lambda / 2 \sigma^{2}, N / 2\right) / \Gamma(N / 2)\right\}$
\end{tabular}

where $K$ is Rician factor, $\bar{\gamma}=h^{2} \mu / \sigma^{2}$ and $h$ is the channel amplitude gain, which is supposed to be constant during $N$ observed samples. 
speaking, in case of discrete received signal the compressed sensing problem could be formulated as [17] [21] [35] [37]:

$$
y=\Phi x=\Phi \Psi s=A s
$$

where $y$ is an $m \times 1$ measurement vector, $\Phi$ is an $m \times N$ measurement or sensing matrix, $\Psi$ is an $N \times N$ sparsity basis matrix, and $s$ is an $N \times 1$ weighting vector. The measurement matrix design is an important issue in CS problem. We seek for stable $\Phi$ to reduce the dimension of $x \in \mathbb{R}^{N}$ to $y \in \mathbb{R}^{m}$ without losing signal information. Also, $\Phi$ requires to be non-adaptive and could not be sparse representation to the columns of $\Psi$ and vice versa. It should be noted that since $m \ll N$, there are infinitely many solutions that satisfy (19) and among all of them we seek for the sparsest solution only. Several reconstruction algorithms have been existed in literature to obtain the optimum solution. Example of these algorithms are Basis Pursuit (BP), Matching Pursuit (MP), Orthogonal Matching Pursuit (OMP), Gradient Pursuit, Stage-wise Orthogonal Matching Pursuit (StOMP), Regularized Orthogonal Matching Pursuit (ROMP), Compressive Sampling Matching Pursuit (CoSaMP), ..., etc., (see [18] [37] and the references therein). The number of measurements required for the signal reconstruction is in the order of $O(m \log N)$. On the other hand, if the signals are analog, then we use analog to information converter (AIC) to extract the information of the signal. Due to that the information rate of a sparse signal is less than its sampling rate. AIC be implemented in different ways, e.g., Wideband Modulation Converter (WMC), multi-coset sampling, multi-rate sampling, and random demodulator [23]. The advantages and disadvantages of the wideband sensing AICs are summarized in Table 4.

Current CS literature is generally centralized on signal reconstruction or approximation while in most communication processes and radars, signals are processed for making detection or classification decision [20] [21]. In detection, we do not ever reconstruct the signal rather than extract sufficient statistics from a small number of random projections or compressive measurements from the frequency band under observation as depicted in Figure 5.

In Figure 5, the PU transmitted signal is detected from the compressed measurements without any reconstruction stage. This generic structure outperforms the conventional structure of detection in two folds: 1) by reducing the computational complexity and 2) guaranteeing better performance with remarkably reduction in the number of measurements due to that the information of $\mathrm{PU}$ is preserved in the compressed measurements. Also, there is no obligation to precise reconstruction of $s$ coefficients in (19). However, the detection decision is accurate because the compressed measurements still contain sufficient data for detection.

By considering the same detection problem as in Section (3.1), however, in compressive detection problem the observing $x$ is changed to $y$ as in (19) where $\Phi \in \mathbb{R}^{m \times N}, m \leq N$ and $\Psi=I_{N \times N}$ is an identity matrix. Hence, the compressive spectrum detection could be formulated as [21]: 
Table 4. Advantages and disadvantages of different wideband sensing AIC approaches.

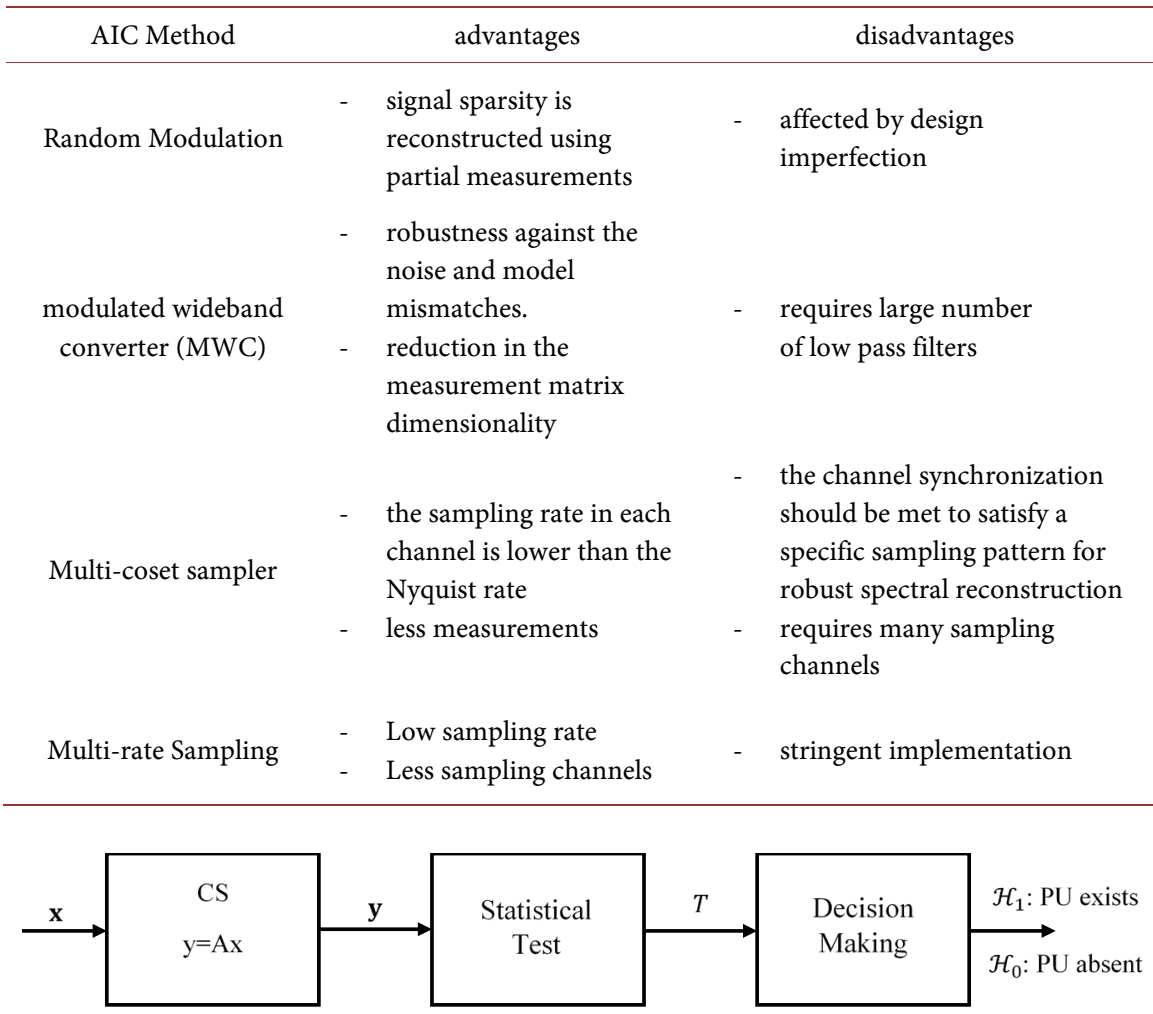

Figure 5. Compressed measurements-based spectrum sensing framework.

$$
y=\left\{\begin{array}{l}
\Phi n: \mathcal{H}_{0} \\
\Phi(x+n): \mathcal{H}_{1}
\end{array}\right.
$$

where $x \in \mathbb{R}^{N \times 1}$ is the PU transmitted signal and $n \sim \mathcal{N}\left(0, \sigma^{2} I_{N}\right)$ is independent and identical distribution (i.i.d.) Gaussian noise. The optimal decision of NP detector is:

$$
T=\frac{f\left(y>\lambda \mid \mathcal{H}_{1}\right)}{f\left(y>\lambda \mid \mathcal{H}_{0}\right)} \underset{\mathcal{H}_{0}}{\stackrel{\mathcal{H}_{1}}{\gtrless}} \lambda
$$

where $\lambda$ is a threshold and the distribution of compressed measurements under $\mathcal{H}_{0}$ and $\mathcal{H}_{1}$ is:

$$
f_{x}(x)=\left\{\begin{array}{l}
\frac{1}{\sqrt{\sigma^{2} \Phi \Phi^{\mathrm{T}}(2 \pi)^{N}}} \exp \left(-y y^{\mathrm{T}} / 2 \sigma^{2} \Phi \Phi^{\mathrm{T}}\right): \mathcal{H}_{0} \\
\frac{1}{\sqrt{\sigma^{2} \Phi \Phi^{\mathrm{T}}(2 \pi)^{N}}} \exp \left(-(y-\Phi x)^{\mathrm{T}}(y-\Phi x) / 2 \sigma^{2} \Phi \Phi^{\mathrm{T}}\right): \mathcal{H}_{1}
\end{array}\right.
$$

Using the same method described in section (3.1) to obtain $P_{F}$ and $P_{D}$ (see (7a) and (7b)) we get:

$$
P_{F}=\mathrm{P}\left(y>\lambda \mid \mathcal{H}_{0}\right)=Q\left(-\frac{\lambda}{\sqrt{x^{\mathrm{T}} \Phi^{\mathrm{T}}\left(\Phi \Phi^{\mathrm{T}}\right)^{-1} \Phi x}}\right)
$$




$$
P_{D}=\mathrm{P}\left(y>\lambda \mid \mathcal{H}_{1}\right)=Q\left(-\frac{\lambda-x^{\mathrm{T}} \Phi^{\mathrm{T}}\left(\Phi \Phi^{\mathrm{T}}\right)^{-1} \Phi x}{\sqrt{x^{\mathrm{T}} \Phi^{\mathrm{T}}\left(\Phi \Phi^{\mathrm{T}}\right)^{-1} \Phi x}}\right)
$$

From (23a), the threshold $\lambda$ required to maximize the $P_{D}$ for specific $P_{F}$ could be computed as:

$$
\lambda=\sqrt{x^{\mathrm{T}} \Phi^{\mathrm{T}}\left(\Phi \Phi^{\mathrm{T}}\right)^{-1} \Phi x} Q^{-1}\left(P_{F}\right)
$$

Let $\Phi \Phi^{\mathrm{T}}=I_{m}$ and substituting (24) for (23-b), we get $\max _{\left(P_{F}, \lambda\right)} P_{D}$ :

$$
\begin{aligned}
P_{D} & =Q\left(Q^{-1}\left(P_{F}\right)-\sqrt{x^{\mathrm{T}} \Phi^{\mathrm{T}}\left(\Phi \Phi^{\mathrm{T}}\right)^{-1} \Phi x} / \sqrt{\sigma^{2}}\right) \\
& =Q\left(Q^{-1}\left(P_{F}\right)-\sqrt{\|\Phi x\|_{2}} / \sqrt{\sigma^{2}}\right)
\end{aligned}
$$

As $m \ll N$, the matrix $\Phi$ is ill-conditioned. For a small controllable perturbation error $\epsilon>0, \Phi$ is enabled to preserve Euclidean length between $x$-sparse vectors. Therefore, $\Phi$ needs to satisfy the restricted isometry property (RIP) [37]. Thus,

$$
(1-\varepsilon) \sqrt{m / N}\|x\|_{2} \leq\|\Phi x\|_{2} \leq(1+\varepsilon) \sqrt{m / N}\|x\|_{2}
$$

Hence, the compressed detector performance is bounded by

$$
P_{D} \approx Q\left(Q^{-1}(P F)-\sqrt{m / N} \sqrt{\gamma}\right)
$$

Next, approaches adopted so far for identifying the spectrum holes under wideband spectrum using CS will be discussed in the following sections.

\section{Compressed Wideband CR Spectrum Detection Approaches}

For years, detecting PU transmitted signal has been acquired from compressed measurements by signal reconstruction [21]. In this section compressed wideband detection approaches will be highlighted. Our discussion will be categorized into two mains categories;

1) Sparse recovery for detection which could be divided into three subcategories: detection-based signal reconstruction, detection based partial signal reconstruction and detection based compressive cooperative.

2) detection based compressed measurements where compressed measurements are used directly to decide whether the PU transmitted signal exists or not on the observed frequency band of interest. Further, compressed detection based cooperative approaches could also comprise the second category as depicted in Figure 6.

\subsection{Detection Based Sparse Signal Recovery}

Prior to the CS introduction as a solution to alleviate sampling rates bottleneck which represents a main challenge for wideband spectrum sensing, it was performed using band-by-band approach. In this case each band has been modulated 


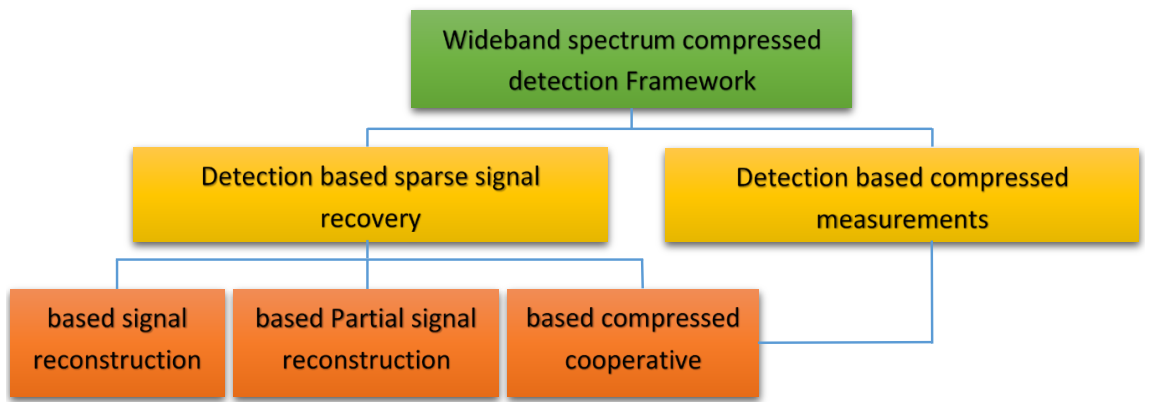

Figure 6. Compressed wideband spectrum detection framework.

to know the locations of these vacant frequency bands. However, this approach of sensing introduces substantially large amount of latency. Later, to beat the latency, filter banks approach was used where each filter is dedicated to scan a specific frequency band. Although filter banks helped on relaxing latency issue introduced by band-by-band method, the number of band pass filters required to achieve sensing purpose was the major problem. On the other hand, wavelet detectors may be suitable solution to overcome filter banks drawbacks, but it may end up with power issue. The nature of the wideband spectrum exhibits sparse structure in open wireless environment in the sense that the number of operative frequency bands is less in comparison to the total number of frequency bands offered by vendors. The sparsity property eases applying CS.

\subsubsection{Detection Based on Signal Reconstruction}

Detection of spectrum holes based on the signal reconstruction has been adopted as the major method used for years before a decision took place. In this method, CR required to build up the spectrum occupancy or PUs transmitted signals using one of the signal reconstruction algorithms [18]. To the best of our knowledge, Tian and Giannakis [38] were the first who built a model to acquire measurement vector depending on Power Spectral Density (PSD) level. It was estimated from the autocorrelation sample sequence, such as high, medium or low to identify spectrum hole. They exploited coarse sensing on analog wideband signal after converted to discrete time sequence using Nyquist sampling rate and then applied CS on the discrete sequence under noiseless channel. PSD gained a great attention where the estimate is acquired from the diagonal matrix of weakly or Wide Sense Stationary (WSS) signals representation in the covariance matrix [39] [40]. Hence, spectrum holes could be identified from the estimated autocorrelation coefficients. Then wavelet edge detector took place to identify edges between adjacent bands to locate the empty bands. In [39] Polo et. al. skip the converting analog to discrete stage and suggested obtaining autocorrelation samples directly from the analog wideband signal unlike [38]. The results in [38] [39] were based on single CR. In [41], a more generalized approach has been introduced based on the autocorrelation vectors obtained from multiple CRs distributing among a network used joint sparse model. However, due to the fact that compressive measurements matrices are not all truly stationary, 
the authors of [42] assumed non WSS signal. The developed algorithm depended on slow channel variation due to small change between two close time instants of the spectrum. In addition, it is focused on monitoring these quite small changing in borders of bands where sparsity found. In this case, the autocorrelation coefficients were estimated from several compressed measurements using random demodulator, however it requires memory support. Eventually, Fourier transform took place to estimate the PSDs.

Other researchers suggested to use the available known information of VHF/UHF TV channels for spectral shape estimate [43] [44] [45], e.g., modulation parameters, channelization, and PU activity. IEEE802.22 WRAN suggested TV channels for CR systems [9]. Bayesian based iterative scheme to estimate a set of occupied bands and their spectral powers simultaneously in wideband spectrum from compressed measurements was proposed in [45]. As well in [43], detecting PU activity was suggested because compressed autocorrelation function computation is conserved. So, PSD of the compressed received signal wouldn't be accessed likens the preset spectral profile. In [45], the sensing task could be further enhanced based on geodesic distance, minimum eigenvalue and by conserving positive semi definite symbol. For this purpose, the difference between the reference spectral profile and auto-correlation matrices sample without reconstructing the signal completely has been used. Even in case of low leakage interference come from other SU, it may increase both the detection performance as in [44]. The signal support is obtained from the over complete dictionary to attain sparsity to elude band by band sweeping. Of course, spectral shape-based detection reduced the sensing time and mitigate interference, i.e., TV bands are sparse in frequency domain. Nonetheless, it requires prior knowledge of interesting signal for comparison with the estimated signal. In other words, memory issue is posed. Cyclostationary feature detection could relax the previous memory with some acceptable complexity as in [46]. Compressive Spectrum Estimator (CSE) was proposed that not only could reconstruct PSD of PU signals from sparse structure by reducing two-dimension cyclic feature. However, it could also have extended to the non-sparse signal to estimate occupancy across a wideband spectrum in CR network. CSE showed robustness against noise uncertainty and interference. Further, the linear relationship between the preferable cyclic feature and the compressed measurements of time-varying cross-correlations used to reconstruct power spectrum was derived in [47]. It rendered the problem to be formulated as convex $\ell_{1}$ minimization solution. Despite these advantages of CSE, the adaptive block size affects the sensing time performance causing the loss of available spectral opportunities.

Undoubtedly, better PSD estimate was achieved exploiting Kronecker matrix as sparsifying basis compared to traditional approaches through substantially reduction of Mean Square Error (MSE) [48]. On the other hand, if sparsity constraints on the power spectrum were relaxed using Least Squares (LSs) and after some rank conditions were hold, blind sampling of power spectrum was pro- 
posed in [49]. Estimating PSDs of the wideband spectrum could be obtained using the cross-correlations between the different outcomes of the sampler. As an extension to the work presented in [46], minimum sparse ruler based multi-coset sampling was used to acquire compressed measurements [49]. It guaranteed unique LS solution when full column rank is met with at least single digit at each column of autocorrelation matrix. In early cooperative approach, Simultaneous Orthogonal Matching Pursuit (SOMP) took place for signal recovery at fusion center [38] [39] [50]. When there is no constraints on sparsity, Simultaneous Sparsity Adaptive Matching Pursuit (SSAMP) was used [51] for more stable reconstruction. When deterioration of spectrum recovery is occurred due to mismatching in standard $\ell_{2} / \ell_{1}$ optimization, the problem could be rendered by dividing the spectrum into blocks with different lengths [52]. Occupancy could be reconstructed using, mixed $\ell_{2} / \ell_{1}$ denoising operator. First, $\ell_{1}$ minimization to each cluster of PSD vector was performed; then $\ell_{2}$ minimization was applied to the aggregation of the minimized cluster by $\ell_{2}$ norm. While Distributed Online Scheme (DOS) uses online distributed least-absolute shrinkage, selection operator Least Absolute Shrinkage and Selection Operator (D-LASSO) algorithm to track the variations on PU's PSDs in ad hoc networks [53]. If the measurements contain complex value, DFT was used as sparsifying basis, Tree based Orthogonal Matching Pursuit (TOMP) would be expedient algorithm [38]. Early mentioned sensing performance is characterized by receiver operating characteristics (ROC) as described in section 3.1 which is a tradeoff between false and detection probabilities, modified blindly optimized compressive sensing (MBOCS) was used in [54]. It used optimized sparsifying basis rather than being fixed. It showed better reconstruction accuracy when compared to simultaneous orthogonal matching pursuit (SOMP) under the same number of measurements. Moreover, when low complexity computations with fast convergence are posed, an iterative technique known as weighted orthogonal matching pursuit (WOMP) [44] was introduced due to its ability rebuild sparsity representation very fast. Following that the optimal sparsifying basis attained by Karhunen-Loéve Transform (KLT) [55] yielded to accuracy, reduction in number of measurements and estimation improvement as [54] due to its coefficients redundancy avoidance in signal representation. Also, it could detect the signal correlation structure and directly update the basis from compressed received signal. In [56], the sensing performance using partially knowledge of the spectrum occupancy has been proposed. The partially knowledge could be provided by remote database, collaborative sensors or any auxiliary entity may deliver that kind of data where only subbands location were known but not their varying PSDs. When signal support is not available, the performance of weighted $\ell_{1}$ minimization is comparable to Modified Basis Pursuit Denoising (MBPDN). It assigned small weights to occupied bands while assigned large weights to the unoccupied bands. It showed some robustness to error when partially knowledge is available. 
It follows that reconstruction algorithm requires having termination point to reduce the sensing period to maximize data throughput when certain satisfaction criterion is met using $\ell_{1}$-norm validation to stop data acquisition as in [56]. Besides, detection performance and computational complexity will be more enhanced and reduced respectively as stated in [57]. The algorithm searches for highly sparse frequency band in the spectrum without a prior knowledge of PU signal by dividing the received wideband spectrum to several frequency band via several bandpass filters followed by AIC. Energy vector is acquired by comparing and averaging these measurements to estimate spectrum occupancy. Also, the choice of sensing matrices, e.g., Discrete Walsh-Hadamard Transform (WHT) and DCT, introduced even far improvement to sensing performance as in [58]. WHT is a matrix that its columns and rows are orthogonal to each other and comprise \pm 1 values only. Decision of compressed detector is thresholding based on centralized chi squared distribution which requires less processing time to unveil spectrum holes. Moreover, WHT and DCT have comparable probability of detection. DCT uses real arithmetic computation due it's lack to complex coefficients and concentrates the energy on the first low order coefficients which make it sparser [59].

The benefits of sparsity led to Sub-Nyquist sampling rate, spectral leakage of spatial, frequency reduction and enhance detection sensitivity. In [53] an arbitrary spatial map of estimating PSDs to identify SHs, but with the expense of battery exhausting of $\mathrm{CR}$ device due to the online monitoring has been created. According to the domain where the signal is sparse, [60] proposed an estimator to just acquire the PSD without reconstruction via solving least squares problem rather than $\ell_{1}$ norm that is used for signal reconstruction. Also by feeding the wideband received signal to a number of filters less in number than the available spectrum bands will reduce the latency caused via band-by-band sensing approach [38] [61]. However exploiting the number of BPF in [61] adds another problem which is how many filters are required. According to the simulation results of [62], it is stated that this number is 12 , which may not be valid in all circumstances.

Although beamforming was not included within IEEE802.22 WRAN framework according to antenna structures [9], CS Minimum Variance Distortion less Response (CSMVDR) algorithm introduced significant reduction in computation complexity at created the reconstruction process. Due to varying the threshold, the probability of detection was improved [62]. Furthermore, spatial beamforming is not only avoiding unnecessary interference to PUs to provide higher throughput for SUs, but it also alleviates the main challenge in traditional methods in estimating the angle of arrival of the signal received by many transmitters. Steven and Sachin [63] designed MAC Spatial Compressed Detection (MAC-SCD) that exploits a spatial degree of freedom as low power wideband detector to estimate spectrum holes efficiently and accurately in CR networks. Also, they used CS to recover angles of arrival by fewer spatial samples obtained 
from less number of antennas than required by traditional one. Ones should keep in mind when the signal of interest of wideband issue is sampled at non-uniform sub-Nyquist sampling rate, approaches such as energy and cyclostationary is no longer valid. So, Aziz et al. [64] formulated the problem of spectrum sensing as missing data problem and proposed Lomb-Scargle method for PSD estimate.

We summarize our discussion in Table 5 to show the advantages and disadvantages of each method.

Table 5. Detection based signal reconstruction methods dis/advantages.

\begin{tabular}{|c|c|c|}
\hline $\begin{array}{l}\text { Article } \\
\text { Ref. }\end{array}$ & Advantages & Disadvantages \\
\hline$[44]$ & $\begin{array}{l}\text { - } \quad \text { elude band by band sweeping } \\
\text { - } \quad \text { reduced the sensing time } \\
\text { - } \quad \text { mitigate interference }\end{array}$ & $\begin{array}{ll} & \text { Computational complexity } \\
\text { - } & \text { requires prior knowledge } \\
\text { - } & \text { memory issue }\end{array}$ \\
\hline$[46]$ & 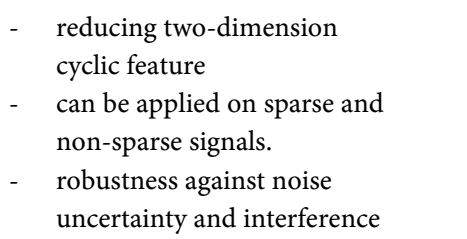 & $\begin{array}{l}\text { - Computational complexity } \\
\text { - block size affects the sensing } \\
\text { time performance }\end{array}$ \\
\hline $\begin{array}{c}{[38][39]} \\
\quad[50]\end{array}$ & $\begin{array}{ll}\text { - } & \text { fast algorithm } \\
\text { - } & \text { easy to implement }\end{array}$ & 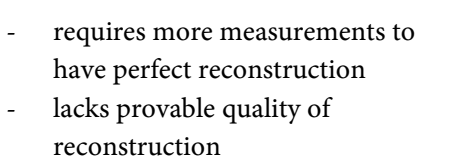 \\
\hline$[50]$ & $\begin{array}{l}\text { - sparsity constraints due to the use } \\
\text { of overcomplete method }\end{array}$ & $\begin{array}{l}\text { the unique solution is } \\
\text { conditioned by complex Gaussian } \\
\text { matrix } M^{2} \geq(2 N-1)^{1} \\
\text { and the selection of } \mathrm{M} \text { is subject to } \\
\text { the auto- and cross } \\
\text { correlation between the row } \\
\text { of sensing matrix used }\end{array}$ \\
\hline$[51]$ & $\begin{array}{l}\text { - } \quad \text { prior sparse level is not required } \\
\text { - } \quad \text { sequential measurements are used } \\
\text { to fasten detection process }\end{array}$ & $\begin{array}{l}\text { - quality of reconstruction } \\
\text { performance is subject to } \\
\text { complexity }\end{array}$ \\
\hline$[52]$ & $\begin{array}{l}\text { using mixed 11/12 norm denoising } \\
\text { operator with LASSO algorithm } \\
\text { increase detection performance. }\end{array}$ & $\begin{array}{l}\text { - require information of the } \\
\text { spectrum boundaries between } \\
\text { different PU as a priori } \\
\text { information }\end{array}$ \\
\hline$[53]$ & $\begin{array}{ll}\text { - } & \text { localized transmitting CRs } \\
\text { - } & \text { sensitive to changes in the system } \\
\text { - } & \text { single hop with low overhead } \\
\text { message between neighbors }\end{array}$ & - battery issue \\
\hline$[56]$ & $\begin{array}{ll}\text { - } & \text { Robust to error } \\
\text { - } & \text { comparable detection performance } \\
\text { compare to MBPDN algorithm }\end{array}$ & $\begin{array}{l}\text { Priori knowledge of } \\
\text { subband locations }\end{array}$ \\
\hline$[57]$ & $\begin{array}{ll}\text { - } & \text { complexity reduction } \\
\text { - } & \text { improved detection accuracy }\end{array}$ & - $\quad$ Priori knowledge is required \\
\hline
\end{tabular}

${ }^{1} M$ and $N$ denote the number rows and columns in the measurement matrix respectively. 


\subsubsection{Detection Based Partial Signal Reconstruction}

Before continuing our discussion of partial signal reconstruction, we need to define what does partial reconstruction-based detection approach mean? It could be defined as an intermediate stage prior to what we discussed in Section 4.1.1 where final decision is declared after signal reconstruction nor does exploiting fewer measurements directly as highlighted in Section 3.2. For this reason, we call it partially signal reconstruction. A multi-resolution Bayesian CS algorithm (BCS) [65] tunes with this concept with fine computational complexity and sensing time reduction whereas the CS problem is inversely solved by Bayesian coefficients obtained by Relevance Vector Machine (VRM). The accuracy of recovered signal is obtained from error bars which it is an indicator for up/down sampling rate according to the varying nature of spectrum occupancy [66]. The error bars strategy is even included in [67].

\subsection{Detection Based Compressed Measurements}

Notwithstanding spectrum occupancy estimate from power spectrum provides more insight on spectrum holes' distribution or spectrum grid. However, in many cases of signal detection problem, we are interested in determining whether the presence of PU transmitted signal on the frequency band under observation or not without ever reconstructing the signal. Thus, compressed measurements-based spectrum detection approach improves sensing into three folds; 1) minimum sensing duration depending on sensing matrices used, 2) increasing CR throughput, and 3) reducing computation cost and implementation complexity. In other words, traditional CS is applied on the received signal firstly. Then reconstruction process took place and after that a decision about existence PU is made as in [38] [53]. In fact, this outcome is obtained with high computation cost and complexity even though the reduction of sampling rate is below the Nyquist rate. The difference between detection based compressed measurements and -signal reconstruction is summarized in Table 6.

Compressed detection or compressed measurements-based detection aims to detect PU transmitted signal without ever reconstruction. Therefore, in [22] direct detection from CS measurements rather than went through reconstruction stage has been proposed. This will increase the CR user data transmission due to the fast detection and the improvement of the sensing time. In [76] the number of measurements required for compressed detection has been specified. Also, as

Table 6. Comparison between two methods of detection based compressed measurements and signal reconstruction.

\begin{tabular}{|c|c|c|}
\hline & detection based compressed measurements & detection based signal reconstruction \\
\hline & $\begin{array}{l}\text { minimum sensing duration depending on } \\
\text { sensing matrices used, }\end{array}$ & - high computation cost and \\
\hline - & increasing CR throughput, and & - $\quad$ high system complexity \\
\hline - & $\begin{array}{l}\text { reducing computation cost and implementa- } \\
\text { tion complexity }\end{array}$ & $\begin{array}{l}\text { - } \begin{array}{l}\text { sensing decision is subject to the used } \\
\text { reconstruction algorithms speed }\end{array}\end{array}$ \\
\hline
\end{tabular}


mentioned earlier, the wireless communication environment exhibits sparsity property by nature. Hence the spectral opportunities that viewed as Markov hidden chain motivated [68] to propose optimum myopic strategy to improve detection performance. The authors of [68] combined CS with online learning of multi-branches bandit, where these branches denote the rows and columns of the sensing matrix, to create an adaptive compressive sensing known as optimum myopic strategy. Nevertheless, the approach of [68] was derived according to an idealistic state of noise free channel with signal support is guaranteed definitely.

Moreover, the optimality of spectrum occupancy decision needs to take into consideration all the previous states of channels occupancy both observation and decision. However, it requires long term of channels activities history database on how it is being taken and left. To enhance the detection performance, in [69] the Algebraic Detection (AD) for compressed wideband spectrum sensing was designed. It exploited the distribution discontinuities method to save the linearity of PU sparse signal by using measurement matrix consists of Dirac function. On the other hand, by relaxing the convex optimization problem used to solve the underdetermined system in estimate compressed wideband spectrum, [70] suggested to design a Nonlinear Least Square Estimator (NLLSE). It is used to unconcealed the SHs directly from few collected samples of multiband signal either correlated or not in time domain. Furthermore, since the noise does not bear any sparsifying basis unlike interference, it has sparsifying basis in some dictionaries. The sparse coefficients positions were used as detector in [67] by measuring the coherence between the sparsity coefficients position on received signal that is free or even corrupted with noise.

\subsection{Detection Based Compressive Cooperative Schemes}

Cooperative compressed spectrum sensing approaches either centralized or distributive CR networks will have a great effect in alleviating challenges related to high sampling rate requirements for wideband spectrum, computation, complexity and power constraint reduction. In addition, noise, and interference issues such as fading, shadowing, hidden node/terminal problem, and network overheads transmission might devastatingly deteriorate spectrum detection performance.

Proceeding in Section 4.2 it has been mentioned that spectrum sensing could be achieved cooperatively either in centralized or distributed scheme. In centralized scheme, the FC collects the sensory information from CR users to determine the decision about spectrum occupancy and broadcasts that result among CR users to be shared opportunistically. While in distributed scheme, a global decision on SHs converge iteratively and jointly through communications performed among CRs. Fading is one of the major challenges in any wireless network which aggravates the detection performance. To tackle this issue, multiple CR terminals cooperate with each other during sensing assignment performing 
spectral estimate locally using data fusion to bring global convergence without the aid of FC to proliferate decision accuracy. Node failure avoidance is obtained by distributive implementation where each SU communicates with its neighboring SUs only using single hop to consume less power throughout sensing mechanism [71]. Another serious issue is the hidden node problem, when an obstacle blocking the CR user from seeing PU signal which lead unintentionally to raise the interference. Albeit the combination of CS with an algebraic detector at FC will provide better detection performance than that obtained with energy detector [69]. However, processing each income from each CR node separately may cause latency unlike if all collected compressed measurements process together at once. A general case of multiple CR users in centralized scheme, rather than the one in [38], is performed in [41] using joint sparsity matrix to improve decision making.

The centralized detection performance [72] could be further improved by formulating spectrum detection problem as support recovery. This improvement could be achieved using several fusion schemes such as decision, quantized data and data fusion in FC with no a prior knowledge of channel state condition and noise statistics. Applying this to the sequential spectrum sensing leads to an increase in the detection sensitivity against noise uncertainty at low SNR using minimum number of measurements. Because sequential detection task is distributed among CR users in the network. On the other hand, Farrag et al. [54] proposed algorithm based on distributed detection matrix targets to increase detection performance of PU with low sensing node complexity among clusters of sensing nodes rather than individuals. In contrast to [72] where each node sends its measurements as a sign vector only to FC. In [54], each sensing node uses its own local detection matrix to sense the wideband spectrum. decision report is generated and sent to FC. In this case, global spectrum occupancy was determined from the aggregation of all received measured signals.

Moreover, sensing node hardware complexity was avoided using upper bound value while maintaining acceptable SNR. Alternatively, in [73] the authors stated that detection could even far improve if sensing problem merged with localization using a grid of available spectrum holes to be easily accessed by SU was created. In other words, the joint framework proposed in [73] might be imagined as a 3D map or grid. It shows the PSD of occupied bands at PU location in the network from sparse observation. For more clarity, just imagine $(x, y, z)$ space where the $x y$-plane is used for PU positioning and $z$-axis represents the occupied bands. The PSD is considered as point in that space or 3D map. As the location of the PU is known to the CR users, the effect hidden node problem could be minimized. However, it requires large storage space to record data or having frequently updated external database. Also, power consumption issue is presented by CR users due to the continuous update. The modified Kronecker joint sparsity matrix in cooperative approach was recommended in [48]. It reduces the mean square error of estimated PSD and maintains the lower sampling 
rate under low compression ratio. In [54] an optimized sparsifying basis has been adopted. The beauty of modified Kronecker comes from the $n$-th dimension, it has. It could represent frequency, time, space and more. This is due to the fact that each dimension exhibits different sparsity representation basis rather than Distributed CS (DCS) which is one-dimension joint sparse matrix. Similarly in [74], Bayesian compressed sensing is used jointly with localization to enhance reconstruction. The PSD map could be estimated using online iterative process. It also yields uncovering the other nearby CR user to minimize the interference caused by CR users themselves [53].

In [40], Ariananda and Leus considered PSD estimation by multiple CR sensors through collected measurements deliver to FC along with Channel State Information (CSI) and sensing matrices to make the final decision about spectrum occupancy. This is for sake of lowering sampling rate per CR sensors cooperated in centralized network in estimate the spectrum state search for spectral opportunities. Later on Romero et al. [75] exploited the model of [40] however, without CSI and instead using cross spectra between the different collected data. In this case, the sensors are grouped, and each group uses different CS structure and sweep different portion of the spectrum; where PSD estimated the correlation of collected measurements. Further reduction on overhead transmission and reports are sent from CR sensor to FC using matrix completion and joint sparsity recovery [76]. Unfortunately, each sensor is equipped with frequency selective filter to sense multiple bands collecting sensory data will encounter latency due to band by band detection. On the other hand, reduction of hardware complexity and sensing time in [77] is a new direction worth-noting. Instead of assigning each CR device with $m$ channels to observe, the $m$ channels will be divided among $m$ CR devices. So each CR device is assigned with only one channel unlike traditional MWC [75]. In this case, $m$ channels in each CR device are adopted to sweep the whole spectrum search for SHs which reduced the sampling rate to be the same as bandwidth of one subband.

\section{Open Research Problems}

\subsection{Compressed Detection of PU Signal at Wideband CR Networks over Fading Channels}

Although the magnificent advantages being gained by introducing CS as a key success for relaxing sampling rate burden at wideband CR networks, the dominant use of CS has been focused almost on signal reconstruction. In literature, there have been several handy reconstruction algorithms used, e.g., basis pursue, greedy algorithm (matching pursue and its extensions),..., etc. In solving detection or sensing problem, we are pivotal interested on deciding if the PU transmitted signal on a frequency band under observation exists or not without ever reconstructing. To the best of our knowledge, this topic of compressed detection or sensing problem isn't attained enough attention yet. Despite the very few published articles to cover this important topic, they ended discussing recon- 
structing the spectrum grid by building the PSD of spectrum occupancy to uncover hidden spectral opportunities location. However, some of these frequency bands may highly likely be occupied by the time for final decision. CS is useful for wide range of statistical inference tasks. Recent published papers dealt compressed detection over AWGN channel, and to the best of our knowledge the topic over several types of fading channels hasn't investigated yet.

\subsection{Minimizing Sensing Time via Wideband Compressed Cooperation CR Networks}

The collaborative approaches have their enormous impact in minimizing sampling rate requirements and computational and hardware complexities per single $\mathrm{CR}$ node and/or to overall CR network performance. In collaborative approach CR users, nodes or sensors agree to form a coalition to detect the hidden WS at wideband spectrum. This coalition could be categorized as centralized or distributed. In distributed mode, spectrum occupancy status on the observed frequency band is converged via a global decision. On the other hand, the final decision on spectrum occupancy status is made by FC as in centralized mode. Furthermore, in both modes, individually or cooperatively, CR users involve sweeping wide range of frequencies sensing WS. The process is performed by dividing the wideband spectrum into $N$ non-overlapping subbands. The process is time consuming which may end to lose the opportunity to use free frequency bands by CR users. However, it is worth noting that the wideband spectrum is sparse in open environment and stressing on this point sensing scenario could be achieved as:

- The spectrum may be divided into sparsity blocks. Let's say $J$ and each CR users or CR group work together by adopting their reception parameters to specific block of interest. And they deliver their sensing compressed measurements to FC for final decision to build the spectrum occupancy.

- Or two different groups of CR may sense the same block to minimize the errors due to noise and interference and send their compressed measurements to FC.

The highlighted point requires an investigation on how this technique will minimizing sensing time and complexity per node and reduce miss detection and false alarm and maximize throughput and detection performance.

\subsection{Optimal Compressed Decision for CR Networks}

As already stated before, spectrum sensing involves the detection of the hidden unused spectral opportunities. However, specifying an optimal threshold for detection is one of the most noteworthy contests. Under optimal requirements, it is required to maintain the probability of false alarm as low as possible and the probability of detection as high as possible as regulated by IEEE802.22 WRAN work group. Minimum false alarm probability improves the utilization of the spectrum, while maximum detection probability reduces the chances of interfe- 
rence due to miss-detection. Miss-detection probability reduction bids protection to the PU against potential CR transmissions. These two metrics is also known as ROC curves where the statistics decision must be adjusted adaptively to satisfy these two opposing requirements for various channel conditions. In most of the published research works, statistics decision is formulated to follow the central chi square distribution with $L$ degrees of freedom under hypothesis $\mathcal{H}_{0}$ and noncentral chi square distribution under hypothesis $\mathcal{H}_{1}$ with $\mu$ is the non-centrality parameter. Since for large number of observation the chi-square distribution could be approximated as Gaussian distribution, the Neyman-Pearson rule may take place to find the optimal threshold for specific false alarm probabilities. A compressed sensing form of threshold is already derived as in [21] at form of Gaussian distribution. An effort to derive an optimal traditional energy detection has been investigated in [78]. However, analytical closed form has not been reached yet under chi-square distribution and more research are required to obtain the closed form optimal threshold for detection compressively as well traditionally. Any breakthrough in this research point will happen in minimizing the sensing time required for detection.

\section{Conclusion}

Spectrum sensing is a mandatory feature to enable self-coexistence for CR in IEEE 802.22 WRAN standardization. Sensing becomes very challenging at wideband $\mathrm{CR}$ network due to the cost of signal processing requirements, i.e., Nyquist sampling rates. Thanks to compressive sensing technique that eases the sampling rates crisis by proving sub-Nyquist sampling and reconstructing the signal using few random projections. It also reduces cost computations, hardware complexity and energy issue. Further, it improved sensing performance over AWGN and fading channels. In this paper compressed spectrum sensing problem at wideband CR networks is addressed and the recent research achievements in solving the problem are discussed.

\section{References}

[1] Force, F.S.P.T. (2002) Report of the Spectrum Efficiency Working Group.

[2] McHenry, M.A., Tenhula, P.A., McCloskey, D., Roberson, D.A. and Hood, C.S. (2006) Chicago Spectrum Occupancy Measurements \& Analysis and a Long-Term Studies Proposal. Proceedings of the 1st International Workshop on Technology and Policy for Accessing Spectrum, Boston, 5 August 2006, 1. https://doi.org/10.1145/1234388.1234389

[3] Zhao, Q. and Sadler, B.M. (2007) A Survey of Dynamic Spectrum Access. Signal Processing Magazine, 24, 79-89. https://doi.org/10.1109/MSP.2007.361604

[4] Berlemann, L. and Mangold, S. (2009) Cognitive Radio and Dynamic Spectrum Access. Wiley Online Library. https://doi.org/10.1002/9780470754429

[5] Lu, L., Zhou, X., Onunkwo, U. and Li, G.Y. (2012) Ten Years of Research in Spectrum Sensing and Sharing in Cognitive Radio. EURASIP Journal on Wireless Communications and Networking, 2012, 28. https://doi.org/10.1186/1687-1499-2012-28 
[6] Yücek, T. and Arslan, H. (2009) A Survey of Spectrum Sensing Algorithms for Cognitive Radio Applications. Communications Surveys \& Tutorials, 11, 116-130. https://doi.org/10.1109/SURV.2009.090109

[7] Akyildiz, I.F., Lee, W.-Y., Vuran, M.C. and Mohanty, S. (2008) A Survey on Spectrum Management in Cognitive Radio Networks. Communications Magazine, 46, 40-48. https://doi.org/10.1109/MCOM.2008.4481339

[8] Akyildiz, I.F., Lee, W.-Y., Vuran, M.C. and Mohanty, S. (2006) Next Generation/Dynamic Spectrum Access/Cognitive Radio Wireless Networks: A Survey. Computer Networks, 50, 2127-2159. https://doi.org/10.1016/j.comnet.2006.05.001

[9] Lei, Z. and Shellhammer, S.J. (2009) IEEE 802.22: The First Cognitive Radio Wireless Regional Area Network Standard. IEEE Communications Magazine, 47, 130-138. https://doi.org/10.1109/MCOM.2009.4752688

[10] Claudino, L. and Abrão, T. (2017) Spectrum Sensing Methods for Cognitive Radio Networks: A Review. Wireless Personal Communications, 95, 5003-5037.

[11] Umar, R. and Sheikh, A.U. (2013) A Comparative Study of Spectrum Awareness Techniques for Cognitive Radio Oriented Wireless Networks. Physical Communication, 9, 148-170. https://doi.org/10.1016/j.phycom.2012.07.005

[12] Akyildiz, I.F., Lo, B.F. and Balakrishnan, R. (2011) Cooperative Spectrum Sensing in Cognitive Radio Networks: A Survey. Physical Communication, 4, 40-62. https://doi.org/10.1016/j.phycom.2010.12.003

[13] Ghasemi, A. and Sousa, E.S. (2006) Impact of User Collaboration on the Performance of Sensing-Based Opportunistic Spectrum Access. Vehicular Technology Conference, Montreal, 25-28 September 2006, 1-6.

[14] Hattab, G. and Ibnkahla, M. (2014) Multiband Spectrum Access: Great Promises for Future Cognitive Radio Networks. Proceedings of the IEEE, 102, 282-306. https://doi.org/10.1109/JPROC.2014.2303977

[15] Janssen, E., Doris, K., Zanikopoulos, A., Murroni, A., Van der Weide, G., Lin, Y., et al. (2013) An 11b 3.6 GS/s Time-Interleaved SAR ADC in 65nm CMOS. Solid-State Circuits Conference Digest of Technical Papers, San Francisco, 17-21 February 2013, 464-465.

[16] Farhang-Boroujeny, B. (2008) Filter Bank Spectrum Sensing for Cognitive Radios. IEEE Transactions on Signal Processing, 56, 1801-1811. https://doi.org/10.1109/TSP.2007.911490

[17] Candè, E.J. and Wakin, M.B. (2008) An Introduction to Compressive Sampling. Signal Processing Magazine, 25, 21-30. https://doi.org/10.1109/MSP.2007.914731

[18] Hayashi, K., Nagahara, M. and Tanaka, T. (2013) A User's Guide to Compressed Sensing for Communications Systems. IEICE Transactions on Communications, 96, 685-712. https://doi.org/10.1587/transcom.E96.B.685

[19] Donoho, D.L. (2006) Compressed Sensing. IEEE Transactions on Information Theory, 52, 1289-1306. https://doi.org/10.1109/TIT.2006.871582

[20] Barkat, M. (2005) Signal Detection and Estimation.

[21] Baraniuk, R.G., Davenport, M.A. and Wakin, M.B. (2006) Detection and Estimation with Compressive Measurements.

[22] Abdelsadek, M.Y., Farrag, M. and Khalaf, T.A. (2014) Compressed Measurements Based Cyclostationary Detector for Wideband Cognitive Radios.

[23] Sun, H., Nallanathan, A., Wang, C.-X. and Chen, Y. (2013) Wideband Spectrum Sensing for Cognitive Radio Networks: A Survey. Wireless Communications, 20, 
74-81. https://doi.org/10.1109/MWC.2013.6507397

[24] Sesham, S. and Sabat, S.L. (2015) Spectrum Sensing for Cognitive Radio Networks. In: White Space Communication, Springer, Berlin, 117-151. https://doi.org/10.1007/978-3-319-08747-4_5

[25] Axell, E., Leus, G., Larsson, E.G. and Poor, H.V. (2012) Spectrum Sensing for Cognitive Radio: State-of-the-Art and Recent Advances. Signal Processing Magazine, 29, 101-116. https://doi.org/10.1109/MSP.2012.2183771

[26] Levy, B.C. (2008) Principles of Signal Detection and Parameter Estimation. Springer Science \& Business Media, Berlin. https://doi.org/10.1007/978-0-387-76544-0

[27] López-Benítez, M. and Casadevall, F. (2012) Improved Energy Detection Spectrum Sensing for Cognitive Radio. IET Communications, 6, 785-796. https://doi.org/10.1049/iet-com.2010.0571

[28] Ibnkahla, M. (2014) Cooperative Cognitive Radio Networks: The Complete Spectrum Cycle. CRC Press, Boca Raton.

[29] Wyglinski, A.M., Nekovee, M. and Hou, T. (2009) Cognitive Radio Communications and Networks: Principles and Practice. Academic Press, Cambridge.

[30] Urkowitz, H. (1967) Energy Detection of Unknown Deterministic Signals. Proceedings of the IEEE, 55, 523-531. https://doi.org/10.1109/PROC.1967.5573

[31] Simon, M.K. and Alouini, M.-S. (2005) Digital Communication over Fading Channels 95. John Wiley \& Sons, Hoboken.

[32] Popovic, H., Stefanovic, D., Mitic, A., Stefanovic, I. and Stefanovic, D. (2007) Some Statistical Characteristics of Nakagami-m Distribution. 8th International Conference on Telecommunications in Modern Satellite, Cable and Broadcasting Services, 26-28 September 2007, 509-512.

[33] Proakis, J.G. (1995) Digital Communications. McGraw-Hill, New York.

[34] Altrad, O. and Muhaidat, S. (2013) A New Mathematical Analysis of the Probability of Detection in Cognitive Radio over Fading Channels. EURASIP Journal on Wireless Communications and Networking, 2013, 159. https://doi.org/10.1186/1687-1499-2013-159

[35] Qaisar, S., Bilal, R.M., Iqbal, W., Naureen, M. and Lee, S. (2013) Compressive Sensing: From Theory to Applications, a Survey. Journal of Communications and Networks, 15, 443-456.

[36] Candes, E. and Romberg, J. (2007) Sparsity and Incoherence in Compressive Sampling. Inverse Problems, 23, 969-985. https://doi.org/10.1088/0266-5611/23/3/008

[37] Foucart, S. and Rauhut, H. (2013) A Mathematical Introduction to Compressive Sensing. Springer, Berlin. https://doi.org/10.1007/978-0-8176-4948-7

[38] Tian, Z. and Giannakis, G.B. (2007) Compressed Sensing for Wideband Cognitive Radios. IEEE International Conference on Acoustics, Speech and Signal Processing, Honolulu, 15-20 April 2007, IV-1357-IV-1360. https://doi.org/10.1109/ICASSP.2007.367330

[39] Polo, Y.L., Wang, Y., Pandharipande, A. and Leus, G. (2009) Compressive Wide-Band Spectrum Sensing. IEEE International Conference on Acoustics, Speech and Signal Processing, Taipei, 19-24 April 2009, 2337-2340. https://doi.org/10.1109/ICASSP.2009.4960089

[40] Ariananda, D.D. and Leus, G. (2012) A Study on Cooperative Compressive Wideband Power Spectrum Sensing. Information Theory in the Benelux and the 2nd Joint WICI IEEE Symposium on Information Theory and Signal Processing in the 
Benelux, Boekelo, 24-25 May 2012, 102-109.

[41] Wang, Y., Pandharipande, A., Polo, Y.L. and Leus, P.G. (2009) Distributed Compressive Wide-Band Spectrum Sensing. Information Theory and Applications Workshop, La Jolla, 8-13 February 2009, 178-183. https://doi.org/10.1109/ITA.2009.5044942

[42] Sundman, D., Chatterjee, S. and Skoglund, M. (2010) On the Use of Compressive Sampling for Wide-Band Spectrum Sensing. IEEE International Symposium on Signal Processing and Information Technology, Luxor, 15-18 December 2010, 354-359. https://doi.org/10.1109/ISSPIT.2010.5711810

[43] Lagunas, E. and Nájar, M. (2013) Compressive Spectrum Sensing Based on Spectral Shape Feature Detection. Proceedings of the 10th International Symposium on Wireless Communication Systems, Ilmenau, 27-30 August 2013, 1-5.

[44] Lagunas, E. and Nájar, M. (2014) Robust Primary User Identification Using Compressive Sampling for Cognitive Radios. IEEE International Conference on Acoustics, Speech and Signal Processing, Florence, 4-9 May 2014, 2347-2351. https://doi.org/10.1109/ICASSP.2014.6854019

[45] Vazquez-Vilar, G., López-Valcarce, R., Mosquera, C. and González-Prelcic, N. (2010) Wideband Spectral Estimation from Compressed Measurements Exploiting Spectral a Priori Information in Cognitive Radio Systems. IEEE International Conference on Acoustics Speech and Signal Processing, Dallas, 14-19 March 2010, 2958-2961.

[46] Leus, G. and Ariananda, D.D. (2011) Power Spectrum Blind Sampling. Signal Processing Letters, 18, 443-446. https://doi.org/10.1109/LSP.2011.2158604

[47] Tian, Z., Tafesse, Y. and Sadler, B.M. (2012) Cyclic Feature Detection with Sub-Nyquist Sampling for Wideband Spectrum Sensing. IEEE Journal of Selected Topics in Signal Processing, 6, 58-69.

[48] Elzanati, A.M., Abdelkader, M.F., Seddik, K.G. and Ghuniem, A.M. (2013) Collaborative Compressive Spectrum Sensing Using Kronecker Sparsifying Basis. IEEE Wireless Communications and Networking Conference, Shanghai, 7-10 April 2013, 2902-2907.

[49] Ariananda, D.D. and Leus, G. (2012) Compressive Wideband Power Spectrum Estimation. IEEE Transactions on Signal Processing, 60, 4775-4789.

https://doi.org/10.1109/TSP.2012.2201153

[50] Ariananda, D.D. and Leus, G. (2011) Wideband Power Spectrum Sensing Using Sub-Nyquist Sampling. 12th International Workshop on Signal Processing Advances in Wireless Communications, San Francisco, 26-29 June 2011, 101-105. https://doi.org/10.1109/SPAWC.2011.5990334

[51] Gu, B. and Yang, Z. (2010) Cooperative Compressed Sensing for Wide-Band Spectrum Detection with Sequential Measurements. 12 th IEEE International Conference on Communication Technology, 11-14 November 2010, 91-95.

[52] Liu, Y. and Wan, Q. (2010) Compressive Wideband Spectrum Sensing for Fixed Frequency Spectrum Allocation.

[53] Bazerque, J.A. and Giannakis, G.B. (2010) Distributed Spectrum Sensing for Cognitive Radio Networks by Exploiting Sparsity. IEEE Transactions on Signal Processing, 58, 1847-1862. https://doi.org/10.1109/TSP.2009.2038417

[54] Farrag, M., Muta, O., El-Khamy, M., Furukawa, H. and El-Sharkawy, M. (2014) Wide-Band Cooperative Compressive Spectrum Sensing for Cognitive Radio Systems Using Distributed Sensing Matrix. 80th Vehicular Technology Conference, 
Vancouver, 14-17 September 2014, 1-6.

[55] Gwon, Y., Kung, H. and Vlah, D. (2012) Compressive Sensing with Optimal Sparsifying Basis and Applications in Spectrum Sensing. Global Communications Conference, Anaheim, 3-7 December 2012, 5386-5391.

[56] Sun, H., Chiu, W.-Y. and Nallanathan, A. (2012) Adaptive Compressive Spectrum Sensing for Wideband Cognitive Radios. Communications Letters, 16, 1812-1815. https://doi.org/10.1109/LCOMM.2012.092812.121648

[57] Alam, S.S., Marcenaro, L. and Regazzoni, C.S. (2013) Enhanced Performance in Wideband Cognitive Radios via Compressive Sensing. Global Conference on Signal and Information Processing, Austin, 3-5 December 2013, 1290-1293.

[58] Alam, S.S., Marcenaro, L. and Regazzoni, C.S. (2014) Impact of Transform Coding in Compressive Sensing Based Wideband Cognitive Radios. 4th International Conference on Wireless Communications, Vehicular Technology, Information Theory and Aerospace \& Electronic Systems, Aalborg, 11-14 May 2014, 1-5. https://doi.org/10.1109/VITAE.2014.6934468

[59] Wang, Y. and Zhang, G. (2014) Compressed Wideband Spectrum Sensing Based on Discrete Cosine Transform. The Scientific World Journal, 2014, Article ID: 464895. https://doi.org/10.1155/2014/464895

[60] Lexa, M.A., Davies, M.E., Thompson, J.S. and Nikolic, J. (2011) Compressive Power Spectral Density Estimation. IEEE International Conference on Acoustics, Speech and Signal Processing, Prague, 22-27 May 2011, 3884-3887.

https://doi.org/10.1109/ICASSP.2011.5947200

[61] Havary-Nassab, V., Hassan, S. and Valaee, S. (2010) Compressive Detection for Wide-Band Spectrum Sensing. International Conference on Acoustics Speech and Signal Processing, Dallas, 15-19 March 2010, 3094-3097.

https://doi.org/10.1109/ICASSP.2010.5496101

[62] Wang, Y., Pandharipande, A. and Leus, G. (2010) Compressive Sampling Based MVDR Spectrum Sensing. 2nd International Workshop on Cognitive Information Processing, Elba, 14-16 June, 2010, 333-337. https://doi.org/10.1109/CIP.2010.5604239

[63] Hong, S. and Katti, S. (2010) Cognitive Spatial Degrees of Freedom Estimation via Compressive Sensing. Proceedings of the 2010 ACM workshop on Cognitive Radio Networks, Chicago, 20 September 2010, 7-12. https://doi.org/10.1145/1859955.1859958

[64] Aziz, B., Traore, S., Nafkha, A. and Le Guennec, D. (2014) Spectrum Sensing for Cognitive Radio Using Multicoset Sampling. Global Communications Conference, Austin, 8-12 December 2014, 816-821. https://doi.org/10.1109/GLOCOM.2014.7036909

[65] Hong, S. (2010) Multi-Resolution Bayesian Compressive Sensing for Cognitive Radio Primary User Detection. Global Telecommunications Conference, Miami, 6-10 December 2010, 1-6.

[66] Hong, S. (2010) Direct Spectrum Sensing from Compressed Measurements. Military Communications Conference, San Jose, 31 October-3 November 2010, 1187-1192. https://doi.org/10.1109/MILCOM.2010.5680103

[67] Liu, B., Fu, P., Meng, S. and Guo, L. (2010) Compressive Sensing Signal Detection Algorithm Based on Location Information of Sparse Coefficients. JDCTA, 4, 79-85. https://doi.org/10.4156/jdcta.vol4.issue8.8

[68] Bagheri, S. and Scaglione, A. (2014) The Cognitive Compressive Sensing Problem. 
IEEE International Symposium on Information Theory, Honolulu, 29 June-4 July 2014, 3195-3199. https://doi.org/10.1109/ISIT.2014.6875424

[69] Moussavinik, H., Guibene, W. and Hayar, A. (2010) Centralized Collaborative Compressed Sensing of Wideband Spectrum for Cognitive Radios. International Congress on UItra Modern Telecommunications and Control Systems and Workshops, Moscow, 18-20 October 2010, 246-252. https://doi.org/10.1109/ICUMT.2010.5676630

[70] Avendi, M., Haghighi, K., Panahi, A. and Viberg, M. (2014) A NLLS Based Sub-Nyquist Rate Spectrum Sensing for Wideband Cognitive Radio.

[71] Zeng, F., Li, C. and Tian, Z. (2011) Distributed Compressive Spectrum Sensing in Cooperative Multihop Cognitive Networks. IEEE Journal of Selected Topics in Signal Processing, 5, 37-48. https://doi.org/10.1109/JSTSP.2010.2055037

[72] Elzanati, A.M., Abdelkader, M.F., Seddik, K.G. and Ghuniem, A.M. (2014) Adaptive Spectrum Hole Detection Using Sequential Compressive Sensing. International Wireless Communications and Mobile Computing Conference, Nicosia, 4-8 August 2014, 1081-1086. https://doi.org/10.1109/IWCMC.2014.6906505

[73] Guibène, W. and Slock, D. (2013) Cooperative Spectrum Sensing and Localization in Cognitive Radio Systems Using Compressed Sensing. Journal of Sensors, 2013, Article ID: 606413. https://doi.org/10.1155/2013/606413

[74] Li, X., Hong, S., Han, Z. and Wu, Z. (2011) Bayesian Compressed Sensing Based Dynamic Joint Spectrum Sensing and Primary User Localization for Dynamic Spectrum Access. Global Telecommunications Conference, Houston, 5-9 December 2011, 1-5.

[75] Mishali, M. and Eldar, Y.C. (2010) From Theory to Practice: Sub-Nyquist Sampling of Sparse Wideband Analog Signals. IEEE Journal of Selected Topics in Signal Processing, 4, 375-391. https://doi.org/10.1109/JSTSP.2010.2042414

[76] Meng, J., Yin, W., Li, H., Hossain, E. and Han, Z. (2011) Collaborative Spectrum Sensing from Sparse Observations in Cognitive Radio Networks. IEEE Journal on Selected Areas in Communications, 29, 327-337. https://doi.org/10.1109/JSAC.2011.110206

[77] Zhang, H., Zhang, Z. and Chau, Y. (2011) Distributed Compressed Wideband Sensing in Cognitive Radio Sensor Networks. IEEE Conference on Computer Communications Workshops, Shanghai, 10-15 April 2011, 13-17.

[78] Taherpour, A., Norouzi, Y., Nasiri-Kenari, M., Jamshidi, A. and Zeinalpour-Yazdi, Z. (2007) Asymptotically Optimum Detection of Primary User in Cognitive Radio Networks. IET Communications, 1, 1138-1145. https://doi.org/10.1049/iet-com:20060645 